\title{
Orthogonally protected glycerols and 2-aminodiols: useful building blocks in heterocyclic chemistry
}

\author{
Anthony Ollivier, ${ }^{\mathrm{a}, \mathrm{b}}$ Marlène Goubert, ${ }^{\mathrm{a}, \mathrm{b}}$ Ahmatjan Tursun,, ${ }^{\mathrm{a}, \mathrm{b}}$ Isabelle Canet, ${ }^{\mathrm{a}, \mathrm{b} *}$ and \\ Marie-Eve Sinibaldi ${ }^{\mathrm{a}}{ }^{\text {b* }}$ \\ ${ }^{a}$ Clermont Université, Université Blaise Pascal, Laboratoire SEESIB, BP 10448, F-63000 \\ Clermont-Ferrand, France \\ ${ }^{b}$ CNRS, UMR 6504, SEESIB, F-63177 Aubière, France \\ E-mail: m-eve.sinibaldi-troin@univ-bpclermont.fr, isabelle.canet@univ-bpclermont.fr
}

\begin{abstract}
The efficient synthesis of orthogonally protected glycerols, 2-aminopropane-1,3-diols and 2aminobutane-1,4-diols that can constitute useful tools in heterocyclic chemistry, is reported. These interesting tri-functionalized small synthons were easily prepared from serine or aspartic acid. In addition, these substrates can be readily transformed into their iodide derivatives in very good yields.
\end{abstract}

Keywords: Amino acids, amino-alcohols, alcohols, amines, halohydrins

\section{Introduction}

Polyfunctionalized small molecules such as glycerols and $\beta$-amino-alcohols are important and versatile units extensively used in the preparation of complex structures like macrolides, ${ }^{1}$ morpholines, ${ }^{2}$ hydroxyamidines ${ }^{3}$ and antibiotics. Moreover, 1,2-diols can serve as precursors for the preparation of vic-halohydrins, useful molecules for synthetic transformations having importance, for example, in the synthesis of halogenated marine products. ${ }^{4}$

Owing to their structures, these building blocks need at first to be protected with groups cleavable independently of the others. Lastly, synthesis of natural compounds required an enantioselective access to these substrates and that can be efficiently investigated starting from readily available natural amino acids.

In connection with our ongoing effort to synthesize spiroketals or analogues, based upon an iterative alkylation of acetone $N, N$-dimethylhydrazone with $\beta$ - and $\gamma$-hydroxyiodides, ${ }^{5}$ we were induced to develop approaches ${ }^{5 \mathrm{c}, \mathrm{d}}$ to several orthogonally protected iodopropanediols, aminopropanols and aminobutanols starting from D-serine or from L-aspartic acid. We describe herein the synthesis and the characterization of orthogonally protected glycerols and 2-aminodiols, together with their efficient conversions into iodides. 


\section{Results and Discussion}

The synthesis of selectively protected $(R)$-iododiols 1a,b,c was accomplished from D-serine in five steps in a range of $20-47 \%$ overall yield (Scheme 1). The first step was a transformation of D-serine into its corresponding alcohol ${ }^{6 a, b}$ followed by esterification of the carboxylic acid to give $(R)-2$ in $83 \%$ yield. ${ }^{6 c}$ In the next step, diol 2 was selectively protected as its pivotal silyl ether $\mathbf{3}^{7}$ in $83 \%$ yield using tert-butyldiphenylsilyl chloride in the presence of imidazole at low temperature.<smiles>N[C@@H](CO)C(=O)O</smiles>

D-serine<smiles>CC(=O)C(COC(C)C)OCC(=O)OCc1ccccc1</smiles>

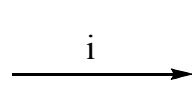
$\stackrel{\text { iii }}{\longleftarrow}$

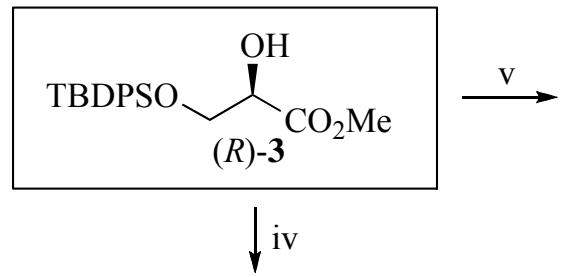<smiles>[M]O[C@@H](CO[SbH2][BH3-])C(=O)OC</smiles>

(R)-4b<smiles>COC(=O)[C@H](O)CO</smiles>

$(R)-2$

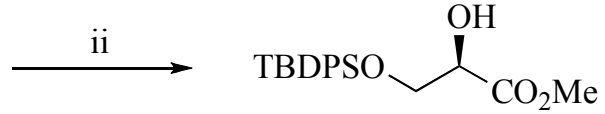

(R)-3<smiles>COC(=O)[C@H]([OH2+])CO[SnH2]CC(C)C</smiles>

<smiles>[CH-]C</smiles><smiles>[R7]C(CO)CO[SbH2][Ba]</smiles>

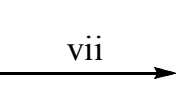<smiles>[R]OC(CI)CO[SbH2][Ba]</smiles>

(S) $-\mathbf{5 a} \mathrm{R}=\mathrm{Bn}$

(S)-5b R = MOM

(R)-1a $\mathrm{R}=\mathrm{Bn}$ (R)-1b R = MOM

$(R)-\mathbf{4 c} \mathrm{R}=\mathrm{TBS}$

(S) $-\mathbf{5 c} \mathrm{R}=\mathrm{TBS}$

$(R)-\mathbf{1 c} \mathrm{R}=\mathrm{TBS}$

Scheme 1. Reagents and conditions : (i) $\mathrm{NaNO}_{2}, \mathrm{H}_{2} \mathrm{SO}_{4}, \mathrm{H}_{2} \mathrm{O}, 5$ days, then $\mathrm{HC}(\mathrm{OMe})_{3}, \mathrm{H}_{2} \mathrm{SO}_{4}, \mathrm{MeOH}$, $\Delta, 30$ min, 83\%; (ii) TBDPSCl, imidazole, $\mathrm{CH}_{2} \mathrm{Cl}_{2},-40{ }^{\circ} \mathrm{C}, 1.5 \mathrm{~h}, 83 \%$; (iii) $\mathrm{BnBr}, \mathrm{Ag}_{2} \mathrm{O}, \mathrm{Et} 2 \mathrm{O}, 20{ }^{\circ} \mathrm{C}$, $82 \%$; (iv) MOMCl, (i-Pr) ${ }_{2} \mathrm{EtN}, \mathrm{CH}_{2} \mathrm{Cl}_{2}, 20{ }^{\circ} \mathrm{C}, 97 \%$; (v) TBSCl, DMAP, imidazole, $\mathrm{CH}_{2} \mathrm{Cl}_{2},-10{ }^{\circ} \mathrm{C}$ then $20{ }^{\circ} \mathrm{C}, 48$ h, 82\%; (vi) $\mathrm{LiBH}_{4}$, THF, $0{ }^{\circ} \mathrm{C}$, $(S)-5 \mathbf{a}(88 \%),(S)-5 \mathbf{b}(88 \%),(S)-5 \mathbf{c}(47 \%)$; (vii) $\mathrm{I}_{2}$, imidazole, $\mathrm{PPh}_{3}, \mathrm{Et}_{2} \mathrm{O} / \mathrm{CH}_{3} \mathrm{CN},-10{ }^{\circ} \mathrm{C}$ to $20^{\circ} \mathrm{C}, \mathbf{1 a}(95 \%), \mathbf{1 b}(73 \%), \mathbf{1 c}(74 \%)$.

We then converted alcohol $\mathbf{3}$ into orthogonally protected derivatives $\mathbf{4 a}, \mathbf{b}, \mathbf{c}$ in good yields using benzyl bromide/silver oxide for $\mathbf{4 a}$, methoxymethyl chloride/diisopropylethylamine for $\mathbf{4} \mathbf{b}^{8}$ and tert-butyldimethylsilyl chloride/dimethylaminopyridine/imidazole for $\mathbf{4 c}$. In the case of $\mathbf{4 b}$, the yield was increased by modifying our previously reported procedure ${ }^{8}$ (see Experimental part). Reduction of methyl esters 4a,b was cleanly achieved with lithium borohydride in tetrahydrofuran at $0{ }^{\circ} \mathrm{C}$ producing the glycerols $\mathbf{5 \mathbf { a } ^ { 9 }}$ and $\mathbf{5 b}$ in $88 \%$ yields; alternatively, $\mathbf{4 c}$ was converted in a moderate yield of $47 \%$ into $5 c$, certainly due to the instability of the tert- 
butyldimethylsilyl group in this series. In a last step, alcohols 5a,b,c were treated with iodine/imidazole in the presence of triphenylphosphine in a mixture of diethyl ether/acetonitrile $(3 / 1)$. Best results were obtained using the following ratio of reactants: 2.4 equiv iodine, 2.2 equiv imidazole, 2.0 equiv triphenylphosphine (see Experimental part), leading almost quantitatively (TLC monitoring) to iodides 1a,b,c (Scheme 1). Compounds 1b,c were found to be slightly unstable during the purification procedure, lowering isolated yields. Moreover, if not used at once, these iodides must be kept at $0{ }^{\circ} \mathrm{C}$ under an inert atmosphere and because of their instability, usually require a filtration before their use in further synthetic transformations.

Protected 2-aminopropane-1,3-diols 10a,b,c and 2-aminobutane-1,4-diols 15a,b,c,d were obtained by a general pathway starting from inexpensive commercial D-serine methyl ester and L-aspartic acid methyl ester, respectively (Scheme 2). In these series, we targeted different protecting groups for the amino function. We chose an oxazolidine moiety (compounds 10c and 15d) in order to allow the simultaneous regeneration of $\beta$-aminoalcohols during the cleavage process. We also utilised the classical tert-butoxycarbonyl protecting group (synthons $\mathbf{1 0 b}$ and 15a). Finally, we tested $N$-diprotection, in which a carbamate was substituted with a benzyl group (compounds 10a and 15c) or a second tert-butoxycarbonyl moiety (compound 15b) with the aim of acquiring, after selective deprotection, either a primary or a secondary amine. To protect the primary alcohol function of all these compounds, we introduced a silyl ether group (tert-butyldiphenylsilyl or tert-butyldimethylsilyl) selectively cleavable later with fluoride ion and stable in the deprotection conditions used for all others groups present in the molecules.

Access to compounds $\mathbf{1 0}$ and $\mathbf{1 5}$ was performed through the reduction of their methyl esters $\mathbf{9}$ and 14. This was carried out using lithium aluminium hydride in tetrahydrofuran for oxazolidines 9c and 14d and using either lithium borohydride in tetrahydrofuran or tetrahydrofuran/ethanol or diisobutylaluminium hydride in ether for all the others compounds 9a,b and 14a,b,c. The polyfunctionalized alcohols 10a,b,c and 15a,b,d thus obtained were then easily iodinated with iodine, triphenylphosphine, imidazole in toluene in the same ratio as that used for the transformation of 5 into 1. For alcohol 15c, activation through its mesylate derivative was required before iodination.

The method of preparation of esters 9 and 14 differed from the nature of the protecting groups of the amino function (Scheme 2).

Thus, $(R)-9$ a was prepared in three steps and 56\% overall yield from D-serine methylester. First, a reductive amination using benzaldehyde and sodium borohydride permitted the nearly quantitative formation of alcohol $(R)-7^{10}$ which was treated with di-tert-butyl dicarbonate to give the carbamate $(R)-8$. The $O$-silylether $(R)$-9a was then isolated after reaction with tertbutyldimethylsilyl chloride/imidazole in anhydrous methylene chloride. Reduction with lithium borohydride furnished alcohol $(S)-10 a$ which was synthezised in four steps and 53\% overall yield from D-serine methylester. Even the iodination seemed to proceed in good yield, as monitored by TLC, we were however unable to isolate 11a because of the instability of the tertbutyldimethylsilyl group during the purification process. 
Ester $(R)-\mathbf{6}^{11}$ was efficiently prepared by protection of D-serine methyl ester as its carbamate and was then transformed either into the tert-butyldiphenylsilyl ether $(R)-\mathbf{9} \mathbf{b}^{12}$ in $\mathbf{9 8 \%}$ yield or into the previously reported oxazolidine $(R)-\mathbf{9 c} .^{13}$ Alcohols $(S)-\mathbf{1 0 b}{ }^{14}$ and $(S)-\mathbf{1 0} \mathbf{c}^{13}$ were finally obtained in three steps and $73 \%$ and $59 \%$ overall yields respectively.<smiles>COC(=O)[C@H](N)CO</smiles>

D-serine methylester
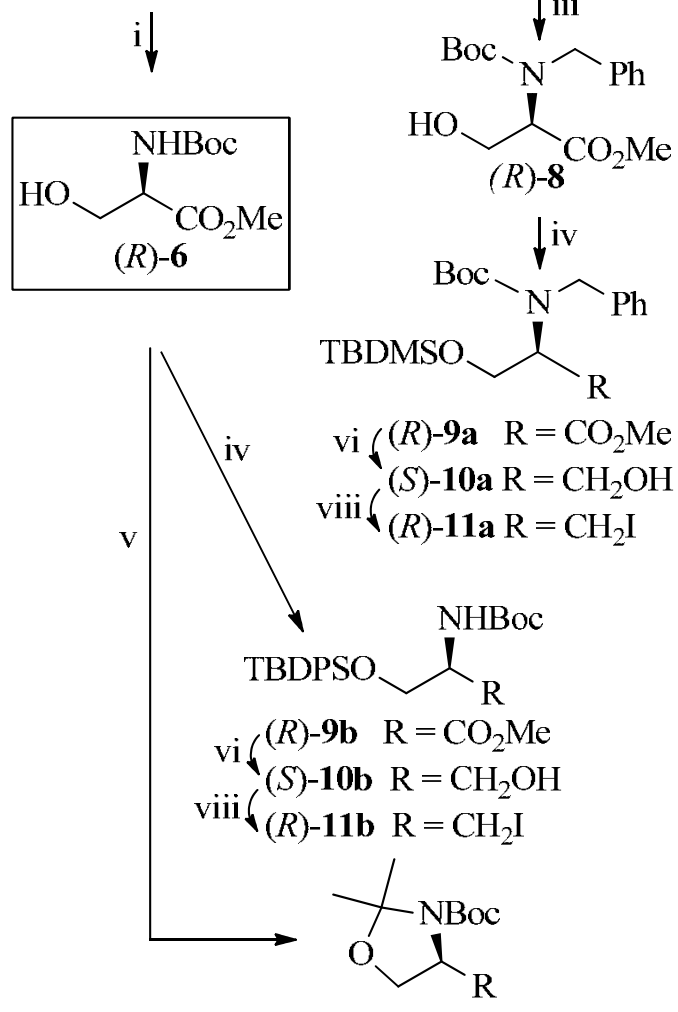

$\operatorname{vii}\left(\begin{array}{l}(R)-9 \mathrm{c} \quad \mathrm{R}=\mathrm{CO}_{2} \mathrm{Me} \\ \operatorname{viii} f(S)-10 \mathrm{c} \mathrm{R}=\mathrm{CH}_{2} \mathrm{OH} \\ (R)-11 \mathrm{c} \mathrm{R}=\mathrm{CH}_{2} \mathrm{I}\end{array}\right.$<smiles>CC(=O)C(CO)NCc1ccccc1</smiles>

(R)-7<smiles>COC(=O)[C@H](CO)N(Cc1ccccc1)C(=O)[C@H](C)C(=O)O</smiles>

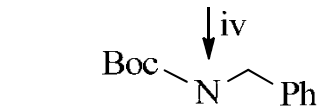


$\mathrm{BrCH}_{2} \mathrm{Ph}, \mathrm{Bu}_{4} \mathrm{NI}$, DMF, 69\%; (xii) MsCl, DMAP, $\mathrm{NEt}_{3}, \mathrm{CH}_{2} \mathrm{Cl}_{2}, 0{ }^{\circ} \mathrm{C}$ then NaI, acetone, $20{ }^{\circ} \mathrm{C}, 12 \mathrm{~h}$, $71 \%$; (xiii) $\mathrm{Boc}_{2} \mathrm{O}$, DMAP, $\mathrm{CH}_{3} \mathrm{CN}, 20^{\circ} \mathrm{C}, 12 \mathrm{~h}, 54 \%$; (xiv) DIBAL-H, Et $2 \mathrm{O},-78^{\circ} \mathrm{C}, 45 \mathrm{~min}, 84 \%$.

Iodination of $(S)$-10b and $(S)$-10c was efficiently achieved using our standard conditions affording the polyfunctionalized substrates $(R)$-11b and $(R)$-11c. These iodo-compounds can be kept several weeks at $0{ }^{\circ} \mathrm{C}$ under argon but needed, as for iodides $\mathbf{1 a}$ and $\mathbf{1 b}$, a filtration on flash silica gel column before use (Scheme 2).

L-Aspartic acid methyl ester was classically transformed into its known carbamate $(S)-\mathbf{1 2} .^{15,16}$ The selective reduction of the carboxylic acid of $(S)-\mathbf{1 2}$ was then realized in two steps by activation with dicyclohexylcarbodiimide/ $N$-hydroxysuccinimide followed by treatment with sodium borohydride, leading to $(S)-\mathbf{1 3}^{17}$ in $79 \%$ yield. Alcohol $(S)-\mathbf{1 3}$ was finally protected giving the pivotal tert-butyldiphenylsilyl ether $(S)$-14a in $98 \%$ yield using tert-butyldiphenylsilyl chloride/imidazole. This latter was further converted to the original esters 14b,c. Indeed, introduction of a second tert-butoxycarbonyl group on 14a was accomplished with di-tert-butyl dicarbonate/dimethylaminopyridine affording $(S)$-14b in 54\% yield. Reaction of the carbamate $(S)$-14a with benzyl bromide in the presence of tetrabutylammonium iodide, furnished $(S)$-14c in $69 \%$ yield.

All these esters were submitted to a reduction process and led finally, from L-aspartic acid methyl ester, to $(S)-15 a$ (four steps, 68\% overall yield), $(S)$-15b and $(S)$-15c (five steps, 31\% and $47 \%$ overall yields, respectively).

Additionally, oxazolidine protection of $(S) \mathbf{- 1 3}$ was accomplished, as reported, ${ }^{15}$ with paratoluenesulfonic acid/2,2-dimethoxypropane in methylene chloride giving $(S)$-14d in $87 \%$ yield, the reduction of which, using lithium aluminium hydride, afforded quantitatively alcohol $(S)$ $\mathbf{1 5 d}^{18}$ (four steps and $61 \%$ overall yield from L-aspartic acid methylester).

In a last step, all the alcohols 15 were transformed into their iodide derivatives $16 \mathbf{a}, 16 \mathbf{b}, 16 \mathbf{c}$ and $\mathbf{1 6} \mathbf{d}^{19}$ in a range of 71-92\% yields using our standard conditions for $\mathbf{1 6 a}, \mathbf{b}, \mathbf{d}$ and through its mesylate for 16c (Scheme 2).

Since compounds 5a, 9c, 10c, 11c, 14d, 15d, 16d were not fully described with regard to their characterisation data, and as some preparative procedures were different to those previously published, we report them in this paper, together with complete sets of data.

\section{Conclusions}

In conclusion, D-serine or L-aspartic acid constitute inexpensive commercially available substrates for the rapid and efficient synthesis of enantiomerically pure orthogonally protected glycerols and 2-aminodiols, applying short synthetic pathways and convenient transformations. Furthermore, these small polyfunctionalized synthons were efficiently transformed into their iodide derivatives. These products constitute useful building blocks for the elaboration of more complex structures. 


\section{Experimental Section}

General. Melting points were measured using a Reichert melting point apparatus and are uncorrected. Infra-Red spectra were recorded on a Perkin-Elmer 881 instrument. Nuclear magnetic resonance spectra were obtained using BRUKER AC 400 spectrometer $\left({ }^{1} \mathrm{H}, 400 \mathrm{MHz}\right.$, ${ }^{13} \mathrm{C}, 100 \mathrm{MHz}$ ). Chemical shifts ( $\delta$ values) are expressed in parts per million (ppm) with solvents as internal standards and coupling constants $(J)$ are expressed in Hertz. Mass spectra were recorded with a Hewlett Packard 5989B instrument and high resolution mass spectra (HRMS) were performed with a Q-TOF micromass. Chromatography was performed using silica gel 60 (230-400 mesh) and thin layer chromatography (TLC) was performed on silica gel $60 \mathrm{PF}_{254}$ plates. Compounds were identified using UV fluorescence $(\lambda=254 \mathrm{~nm})$ and/or staining with a $5 \%$ phosphomolybdic acid solution in ethanol following by heating. Commercially reagents were used as received from the manufacturers except for tetrahydrofuran (distilled from potassium/benzophenone) and dichloromethane (dried over calcium hydride prior to use).

\section{General procedure for the preparation of iodides}

To a stirred solution of the appropriate alcohol $(1.02 \mathrm{mmol})$ in a $3 / 2$ mixture of diethyl ether/acetonitrile $(15 \mathrm{~mL})$ at $0{ }^{\circ} \mathrm{C}$ or in toluene $(15 \mathrm{~mL})$ at $20{ }^{\circ} \mathrm{C}$ were successively added imidazole $(2.25 \mathrm{mmol})$, iodine $(2.45 \mathrm{mmol})$ and triphenylphosphine $(2.04 \mathrm{mmol})$. The stirring was maintained for $1 \mathrm{~h}$ at $0{ }^{\circ} \mathrm{C}$ and then for $24 \mathrm{~h}$ at $20{ }^{\circ} \mathrm{C}$ or directly at $20{ }^{\circ} \mathrm{C}$ for $24 \mathrm{~h}$ when toluene was used. The reaction was quenched by addition of a $10 \%$ aqueous sodium thiosulfate solution $(6 \mathrm{~mL})$ followed by saturated aqueous ammonium chloride solution $(3 \mathrm{~mL})$. The organic layer was extracted with ether, washed with a $10 \%$ aqueous sodium thiosulfate solution until the solution became colourless. Then it was washed with brine, dried $\left(\mathrm{MgSO}_{4}\right)$ and concentrated under reduced pressure. The residue was purified by flash column chromatography using as solvents a mixture of ethyl acetate/cyclohexane to afford the corresponding iodide.

\section{General procedure for reduction of esters: method A with $\mathrm{LiBH}_{4}$ in THF}

To a stirred solution of the appropriate ester $(1.04 \mathrm{mmol})$ in anhydrous tetrahydrofuran $(20 \mathrm{~mL})$, at $0{ }^{\circ} \mathrm{C}$ and under argon atmosphere, was dropwise added a $2.0 \mathrm{M}$ lithium borohydride solution $(1.56 \mathrm{mmol})$ in tetrahydrofuran. The resulting mixture was stirred for $12 \mathrm{~h}$ at $20{ }^{\circ} \mathrm{C}$, quenched by addition of saturated aqueous ammonium chloride solution $(1.0 \mathrm{~mL})$. After extraction with ethyl acetate, the organic layer was washed with brine, dried $\left(\mathrm{MgSO}_{4}\right)$ and concentrated under reduced pressure. The residue was then purified by flash column chromatography (ethyl acetate/cyclohexane) to afford the corresponding alcohol.

\section{General procedure for reduction of esters: method B with $\mathrm{LiBH}_{4}$ in THF/EtOH}


To a stirred solution of ester $(1.56 \mathrm{mmol})$ in tetrahydrofuran/ethanol (v/v $1 / 9)$, at $0{ }^{\circ} \mathrm{C}$ and under argon atmosphere was dropwise added a $2.0 \mathrm{M}$ lithium borohydride solution $(0.78 \mathrm{mmol})$ in tetrahydrofuran. The resulting mixture was stirred for $12 \mathrm{~h}$ at $20^{\circ} \mathrm{C}$, and the reaction was stopped by addition of a $0.5 \mathrm{M}$ hydrochloric acid solution. After extraction with diethyl ether, the organic layer was washed with saturated aqueous sodium bicarbonate solution, followed by brine, dried $\left(\mathrm{MgSO}_{4}\right)$ and concentrated under reduced pressure. The residue was then purified by flash column chromatography (ethyl acetate/cyclohexane) to afford the corresponding alcohol.

\section{General procedure for reduction of esters: method C with $\mathrm{LiAlH}_{4}$ in THF}

To a stirred solution of ester $(1.94 \mathrm{mmol})$ in anhydrous tetrahydrofuran $(5 \mathrm{~mL})$, at $0{ }^{\circ} \mathrm{C}$ and under argon atmosphere, was dropwise added a $1.0 \mathrm{M}$ lithium aluminium hydride solution in tetrahydrofuran $(1.16 \mathrm{mmol})$. The resulting mixture was stirred for $12 \mathrm{~h}$ at $20^{\circ} \mathrm{C}$. The reaction was quenched at $0{ }^{\circ} \mathrm{C}$ by sequential addition of water, $15 \%$ sodium hydroxide solution and water. The resulting mixture was dried $\left(\mathrm{MgSO}_{4}\right)$, filtered and the solvent was concentrated under reduced pressure to afford the corresponding pure alcohol.

\section{General procedure for preparation of oxazolidines}

To a solution of aminoalcohol $(3.47 \mathrm{mmol})$ in anhydrous dichloromethane $(40 \mathrm{~mL})$ at $20{ }^{\circ} \mathrm{C}$, was added para-toluenesulfonic acid (60 $\mathrm{mg}, 3.12 \mathrm{mmol}$ ) followed by 2,2-dimethoxypropane (1.29 $\mathrm{mL}, 10.4 \mathrm{mmol}$ ). The mixture was stirred $48 \mathrm{~h}$ at $20{ }^{\circ} \mathrm{C}$ before neutralization by sodium

carbonate. After filtration on a Celite ${ }^{\circledR}$ pad, the solvent was removed under reduced pressure and the residue purified by flash column chromatography (ethyl acetate/cyclohexane, 3:7).

(2R)-2-(Benzyloxy)-3-(tert-butyldiphenylsilyloxy)-1-iodopropane (1a). Compound 1a was prepared from alcohol $(S)$-5a in diethyl ether/acetonitrile following the general procedure for the preparation of iodides. Pale yellow oil (515 mg, 95\%); $R_{f} 0.89$ (EtOAc/cyclohexane, $1 / 19$ ); $[\alpha]_{\mathrm{D}}{ }^{25}$ $=-9.24\left(\mathrm{c} 0.99, \mathrm{CHCl}_{3}\right)$; IR (neat) $v_{\max } 2929,2857,2360,2344,1472,1456,1427,1188,1113$; ${ }^{1} \mathrm{H}$ NMR $\left(\mathrm{CDCl}_{3}\right) \delta 7.72-7.68(\mathrm{~m}, 4 \mathrm{H}, \mathrm{H}-\mathrm{Ar}), 7.46-7.30(\mathrm{~m}, 11 \mathrm{H}, \mathrm{H}-\mathrm{Ar}), 4.62(\mathrm{~d}, 1 \mathrm{H}, J=12.5$ $\left.\mathrm{Hz}, \mathrm{CH}_{2} \mathrm{Ph}\right), 4.55\left(\mathrm{~d}, 1 \mathrm{H}, J=12.5 \mathrm{~Hz}, \mathrm{CH}_{2} \mathrm{Ph}\right), 3.82\left(\mathrm{dd}, 1 \mathrm{H}, J=10.5,5.0 \mathrm{~Hz}, \mathrm{CH}_{2}-\mathrm{O}\right), 3.74$ (dd, $\left.1 \mathrm{H}, J=10.5,5.5 \mathrm{~Hz}, \mathrm{CH}_{2}-\mathrm{O}\right), 3.52-3.40\left(\mathrm{~m}, 3 \mathrm{H}, \mathrm{CH}_{2}-\mathrm{I}\right.$ and $\left.\mathrm{CH}-\mathrm{O}\right), 1.09(\mathrm{~s}, 9 \mathrm{H}, t-\mathrm{Bu}) ;{ }^{13} \mathrm{C} \mathrm{NMR}$ $\left(\mathrm{CDCl}_{3}\right) \delta 138.1$ (C-Ar), 135.8 (C-Ar), 133.4 (C-Ar), 130.0 (C-Ar), 128.5 (C-Ar), 127.9 (C-Ar), $78.5(\mathrm{CH}-\mathrm{O}), 72.1\left(\mathrm{CH}_{2} \mathrm{Ph}\right), 65.1\left(\mathrm{CH}_{2}-\mathrm{O}\right), 27.0(t-\mathrm{Bu}), 19.9(\mathrm{C}), 7.0\left(\mathrm{CH}_{2}-\mathrm{I}\right)$; HRMS-ESI $\mathrm{m} / z$ calcd for $\mathrm{C}_{26} \mathrm{H}_{311} \mathrm{O}_{2} \mathrm{SiINa}[\mathrm{M}+\mathrm{Na}]^{+}$: 553.1036, found : 553.1014 .

(2R)-3-(tert-Butyldiphenylsilyloxy)-1-iodo-2-(methoxymethoxy)propane (1b). Compound 1b was prepared from alcohol $(S)$-5b in diethyl ether/acetonitrile following the general procedure for the preparation of iodides. Pale yellow oil (360 mg, 73\%); $R_{f} 0.90$ (EtOAc/cyclohexane, 1/1); $[\alpha]_{\mathrm{D}}^{25}=-7.9\left(\mathrm{c} 1.03, \mathrm{CHCl}_{3}\right)$; IR (neat) $v_{\max } 2954,2931,2858,2360,2343,1473,1428,1112$; ${ }^{1} \mathrm{H}$ NMR $\left(\mathrm{CDCl}_{3}\right) \delta$ 7.70-7.65 (m, 4H, H-Ar), 7.44-7.37 (m, 6H, H-Ar), 4.66 (s, 2H, O-CH $2-\mathrm{O}$ ), 3.77 (dd, $1 \mathrm{H}, J=10.5,5.0 \mathrm{~Hz}, \mathrm{CH}_{2}-\mathrm{O}$ ), 3.68 (dd, $1 \mathrm{H}, J=10.5,5.0 \mathrm{~Hz}, \mathrm{CH}_{2}-\mathrm{O}$ ), 3.58 (quint, $1 \mathrm{H}$, $J=5.0 \mathrm{~Hz}, \mathrm{CH}-\mathrm{O}$ ), 3.47 (dd, $\left.1 \mathrm{H}, J=10.5,5.0 \mathrm{~Hz}, \mathrm{CH}_{2}-\mathrm{I}\right), 3.39$ (dd, $1 \mathrm{H}, J=10.5,5.0 \mathrm{~Hz}, \mathrm{CH}_{2}-$ I), $3.25\left(\mathrm{~s}, 3 \mathrm{H}, \mathrm{OCH}_{3}\right), 1.06(\mathrm{~s}, 9 \mathrm{H}, t-\mathrm{Bu}) ;{ }^{13} \mathrm{C} \mathrm{NMR}\left(\mathrm{CDCl}_{3}\right) \delta 135.7(\mathrm{C}-\mathrm{Ar}), 133.2(\mathrm{C}-\mathrm{Ar})$ 
129.8 (C-Ar), 127.8 (C-Ar), $96.0\left(\mathrm{O}-\mathrm{CH}_{2}-\mathrm{O}\right), 76.5(\mathrm{CHO}), 65.3\left(\mathrm{CH}_{2}-\mathrm{O}\right), 55.9\left(\mathrm{OCH}_{3}\right), 26.8(t-$ $\mathrm{Bu}), 19.2(\mathrm{C}), 7.4\left(\mathrm{CH}_{2}-\mathrm{I}\right)$; HRMS-ESI $m / z$ calcd for $\mathrm{C}_{21} \mathrm{H}_{30} \mathrm{O}_{4} \mathrm{SiNa}[\mathrm{M}+\mathrm{Na}]^{+}: 507.0828$, found : 507.0836 .

(2R)-2-(tert-Butyldimethylsilyloxy)-3-(tert-butyldiphenylsilyloxy)-1-iodopropane (1c). Compound 1c was prepared from alcohol $(S)$-5c in diethyl ether/acetonitrile following the general procedure for the preparation of iodides. Pale yellow oil (415 mg, 74\%); $R_{f} 0.90$ (EtOAc/cyclohexane, 1/9); IR (neat) $v_{\max } 2956,2929,2858,2360,2343,1473,1428,1126,1112,1107 ;{ }^{1} \mathrm{H}$ NMR $\left(\mathrm{CDCl}_{3}\right) \delta$ 7.70-7.65 (m, 4H, H-Ar), 7.47-7.37 (m, 6H, H-Ar), 3.63 (m, 1H, CHO), 3.58-3.53 (m, 2H, $\mathrm{CH}_{2}-$ O), 3.47 (dd, $\left.1 \mathrm{H}, J=10.0,4.0 \mathrm{~Hz}, \mathrm{CH}_{2}-\mathrm{I}\right), 3.36$ (dd, $\left.1 \mathrm{H}, J=10.0,4.0 \mathrm{~Hz}, \mathrm{CH}_{2}-\mathrm{I}\right), 1.06$ (s, 9H, $t$ $\mathrm{Bu}), 0.85(\mathrm{~s}, 9 \mathrm{H}, t \mathrm{Bu}), 0.06\left(\mathrm{~s}, 3 \mathrm{H}, \mathrm{CH}_{3}\right),-0.06\left(\mathrm{~s}, 3 \mathrm{H}, \mathrm{CH}_{3}\right) ;{ }^{13} \mathrm{C} \mathrm{NMR}\left(\mathrm{CDCl}_{3}\right) \delta 135.9(\mathrm{C}-$ Ar), 135.8 (C-Ar), 135.6 (C-Ar), 135.5 (C-Ar), 133.3 (C-Ar), 133.2 (C-Ar), 129.8 (C-Ar), 127.7 (C-Ar), $71.7(\mathrm{CH}-\mathrm{O}), 66.8\left(\mathrm{CH}_{2}-\mathrm{O}\right), 26.9(t-\mathrm{Bu}), 25.8(t-\mathrm{Bu}), 19.2(\mathrm{C}), 18.0(\mathrm{C}), 11.6\left(\mathrm{CH}_{2}-\mathrm{I}\right),-$ 4.6 $\left(\mathrm{CH}_{3}\right)$, - $4.7\left(\mathrm{CH}_{3}\right)$; HRMS-ESI $m / z$ calcd for $\mathrm{C}_{25} \mathrm{H}_{39} \mathrm{O}_{2} \mathrm{Si}_{2} \mathrm{INa}[\mathrm{M}+\mathrm{Na}]^{+}: 577.1431$, found : 577.1432 .

Methyl (2R)-2-(benzyloxy)-3-(tert-butyldiphenylsilyloxy)propanoate (4a). To a stirred solution of $(R)-3(2.80 \mathrm{~g}, 7.83 \mathrm{mmol})$ in anhydrous diethyl ether $(35 \mathrm{~mL})$, at $20{ }^{\circ} \mathrm{C}$ and under argon atmosphere, were added silver oxide (I) $(2.72 \mathrm{~g}, 11.75 \mathrm{mmol})$ followed by benzyl bromide $(2.33 \mathrm{~mL}, 19.58 \mathrm{mmol})$. The reaction mixture was stirred at $20{ }^{\circ} \mathrm{C}$ for further 3 days. After filtration on a Celite ${ }^{\circledR}$ pad, the filtrate was concentrated. The residue was purified by flash column chromatography (ethyl acetate/cyclohexane, 1:19) to afford 4a (2.88 g, 82\%) as a colorless oil; $R_{f} 0.82($ EtOAc/cyclohexane, $1 / 1) ;[\alpha]_{\mathrm{D}}{ }^{25}=+37.09\left(\mathrm{c} 0.86, \mathrm{CHCl}_{3}\right)$; IR (neat) $v_{\max }$ 2954, 2932, 2858, 1749, 1428; ${ }^{1} \mathrm{H}$ NMR $\left(\mathrm{CDCl}_{3}\right) \delta 7.68-7.61(\mathrm{~m}, 4 \mathrm{H}, \mathrm{H}-\mathrm{Ar}), 7.36-7.21(\mathrm{~m}, 11 \mathrm{H}$, H-Ar), 4.68 (d, $\left.1 \mathrm{H}, J=12.0 \mathrm{~Hz}, \mathrm{CH}_{2} \mathrm{Ph}\right), 4.45\left(\mathrm{~d}, 1 \mathrm{H}, J=12.0 \mathrm{~Hz}, \mathrm{CH}_{2} \mathrm{Ph}\right), 4.06$ (t, $1 \mathrm{H}, J=5.0$ $\mathrm{Hz}, \mathrm{CH}-\mathrm{O}$ ), 3.88 (dd, $1 \mathrm{H}, J=10.5,5.5 \mathrm{~Hz}, \mathrm{CH}_{2}-\mathrm{O}$ ), 3.86 (dd, $1 \mathrm{H}, J=10.5,5.5 \mathrm{~Hz}, \mathrm{CH}_{2}-\mathrm{O}$ ), 3.67 (s, 3H, $\left.\mathrm{OCH}_{3}\right), 0.96(\mathrm{~s}, 9 \mathrm{H}, t-\mathrm{Bu}) ;{ }^{13} \mathrm{C} \mathrm{NMR}\left(\mathrm{CDCl}_{3}\right) \delta 171.4\left(\mathrm{CO}_{2} \mathrm{Me}\right), 137.5$ (C-Ar), $135.7(\mathrm{C}-$ Ar), 133.2 (C-Ar), 129.7 (C-Ar), 128.4 (C-Ar), 127.7 (C-Ar), 79.3 (CH-O), $72.5\left(\mathrm{CH}_{2} \mathrm{Ph}\right), 64.8$ $\left(\mathrm{CH}_{2}-\mathrm{O}\right), 51.9\left(\mathrm{OCH}_{3}\right), 26.7(t-\mathrm{Bu}), 19.2(\mathrm{C})$; HRMS-ESI $m / z$ calcd for $\mathrm{C}_{27} \mathrm{H}_{32} \mathrm{O}_{4} \mathrm{SiNa}[\mathrm{M}+$ $\mathrm{Na}]^{+}: 471.1968$, found: 471.1963 .

Methyl (2R)-3-(tert-butyldiphenylsilyloxy)-2-(methoxymethoxy)propanoate (4b). Diiso propylethylamine $(1.97 \mathrm{~mL}, 11.43 \mathrm{mmol})$ followed $15 \mathrm{~min}$ later by methoxymethane chloride $(0,91 \mathrm{~mL}, 11.43 \mathrm{mmol})$ were added under argon atmosphere to a stirred solution of $(R)-3(1.37 \mathrm{~g}$, $3.81 \mathrm{mmol})$ in anhydrous dichloromethane $(10 \mathrm{~mL})$. The resulting mixture was kept at $20^{\circ} \mathrm{C}$ for $12 \mathrm{~h}$ before additional diisopropylethylamine $(0.68 \mathrm{~mL}, 3.81 \mathrm{mmol})$ and methoxymethane chloride $(0,30 \mathrm{~mL}, 3.81 \mathrm{mmol})$ were added followed, one more time, $6 \mathrm{~h}$ later, by the addition of the same amounts of the two reagents. After $48 \mathrm{~h}$, the reaction was quenched by water $(2.5 \mathrm{~mL})$. The layers were separated and the organic one was washed with water and dried $\left(\mathrm{MgSO}_{4}\right)$ before concentration to afford $\mathbf{4 b}$ as a pale oil $(1.484 \mathrm{~g}, 97 \%) ; R_{f} 0.51$ (EtOAc/cyclohexane, $\left.1 / 4\right) ;[\alpha]_{\mathrm{D}}{ }^{25}$ $=+7.0\left(\mathrm{c} 1.3, \mathrm{CHCl}_{3}\right)$. For details of ${ }^{1} \mathrm{H}$ and ${ }^{13} \mathrm{C}$ NMR spectroscopic data, see our previously reported work described in reference 8 . 
Methyl (2R)-2-(tert-butyldimethylsilyloxy)-3-(tert-butyldiphenylsilyloxy)propanoate (4c). 4-Dimethylaminopyridine $(0.034 \mathrm{~g}, 0.28 \mathrm{mmol})$ and imidazole $(0.105 \mathrm{~g}, 1.54 \mathrm{mmol})$ were added consecutively at $-10{ }^{\circ} \mathrm{C}$ under argon atmosphere to a stirred solution of $(R)-3(0.500 \mathrm{~g}, 1.39$ $\mathrm{mmol})$ in anhydrous dichloromethane $(10 \mathrm{~mL})$. The mixture was stirred for $15 \mathrm{~min}$ before adding tert-butyldimethylsilyl chloride $(0.232 \mathrm{~g}, 1.54 \mathrm{mmol})$ and the stirring was pursued $48 \mathrm{~h}$. The reaction was stopped by adjunction of water $(4 \mathrm{~mL})$ and the resulting solution was extracted with ethyl acetate. The organic layer was washed with brine and dried $\left(\mathrm{MgSO}_{4}\right)$. Solvent evaporation afforded after purification by flash chromatography (ether/pentane; 1:19) 4c as an oil (0.540 g, $82 \%$ ), $R_{f} 0.74$ (ether/pentane, 1/19); $[\alpha]_{\mathrm{D}}{ }^{25}=+0.80$ (c 1.12, $\mathrm{CHCl}_{3}$ ); IR (neat) $v_{\max } 2953,2931$, 2858, 1759, 1473, 1428, 1151, 1113; ${ }^{1} \mathrm{H} \mathrm{NMR}\left(\mathrm{CDCl}_{3}\right) \delta 7.61-7.59$ (m, 4H, H-Ar), 7.35-7.28 (m, 6H, H-Ar), 4.25 (t, $1 \mathrm{H}, J=5.0 \mathrm{~Hz}, \mathrm{CH}-\mathrm{O}), 3.75$ (bd, $\left.2 \mathrm{H}, J=5.0 \mathrm{~Hz}, \mathrm{CH}_{2}-\mathrm{O}\right), 3.65$ (s, 3H, O$\left.\mathrm{CH}_{3}\right), 0.95(\mathrm{~s}, 9 \mathrm{H}, t \mathrm{Bu}), 0.82(\mathrm{~s}, 9 \mathrm{H}, t-\mathrm{Bu}), 0.00\left(\mathrm{~s}, 3 \mathrm{H}, \mathrm{CH}_{3}\right),-0.03\left(\mathrm{~s}, 3 \mathrm{H}, \mathrm{CH}_{3}\right) ;{ }^{13} \mathrm{C} \mathrm{NMR}$ $\left(\mathrm{CDCl}_{3}\right) \delta 172.5\left(\mathrm{CO}_{2} \mathrm{Me}\right), 135.6$ (C-Ar), 135.5 (C-Ar), 133.3 (C-Ar), 133.1 (C-Ar), 129.7 (C$\mathrm{Ar}), 127.7$ (C-Ar), $73.7(\mathrm{CH}-\mathrm{O}), 66.5\left(\mathrm{CH}_{2}-\mathrm{O}\right), 51.8\left(\mathrm{O}-\mathrm{CH}_{3}\right), 26.6(t-\mathrm{Bu}), 25.7(t-\mathrm{Bu}), 19.2(\mathrm{C})$, -5.2 $\left(\mathrm{CH}_{3}\right)$; HRMS-ESI $\mathrm{m} / z$ calcd for $\mathrm{C}_{26} \mathrm{H}_{40} \mathrm{O}_{4} \mathrm{Si}_{2} \mathrm{Na}[\mathrm{M}+\mathrm{Na}]^{+}: 495.2363$; found: 495.2351 .

(2S)-2-(Benzyloxy)-3-(tert-butyldiphenylsilyloxy)propanol (5a). Compound 5a was obtained from reduction of $(R)-4 a$ using the general Method A of reduction. Oil $(0.384 \mathrm{~g}, 88 \%) ; R_{f} 0.55$ (EtOAc/cyclohexane, 1/4); $[\alpha]_{\mathrm{D}}{ }^{25}=-24.1\left(\mathrm{c} 1.7, \mathrm{CHCl}_{3}\right)\left(\mathrm{lit}^{9}[\alpha]_{\mathrm{D}}{ }^{25}=-24.5\left(\mathrm{c} \mathrm{1.1}, \mathrm{CHCl}_{3}\right)\right.$; IR (neat) $v_{\max } 3445,3069,1472,1427,1113,1106 ;{ }^{1} \mathrm{H} \mathrm{NMR}\left(\mathrm{CDCl}_{3}\right) \delta 7.70-7.68(\mathrm{~m}, 4 \mathrm{H}, \mathrm{H}-\mathrm{Ar})$, 7.45-7.29 (m, 11H, H-Ar), 4.65 (d, 1H, $\left.J=11.5 \mathrm{~Hz}, \mathrm{CH}_{2} \mathrm{Ph}\right), 4.53$ (d, 1H, $J=11.5 \mathrm{~Hz}, \mathrm{CH}_{2} \mathrm{Ph}$ ), 3.84-3.62 (m, 5H, $\left.\mathrm{CH}_{2}-\mathrm{O}, \mathrm{CH}-\mathrm{O}, \mathrm{CH}_{2}-\mathrm{OH}\right), 2.09$ (dd, 1H, $\left.J=6.5,5.5 \mathrm{~Hz}, \mathrm{OH}\right), 1.08$ (s, 9H, $t$ $\mathrm{Bu}) ;{ }^{13} \mathrm{C}$ NMR $\left(\mathrm{CDCl}_{3}\right) \delta 138.3$ (C-Ar), 135.6 (C-Ar), 133.2 (C-Ar), 129.8 (C-Ar), $128.5(\mathrm{C}-\mathrm{Ar})$, 127.8 (C-Ar), $79.6(\mathrm{CH}-\mathrm{O}), 72.2\left(\mathrm{CH}_{2}\right), 63.6\left(\mathrm{CH}_{2}\right), 62.8\left(\mathrm{CH}_{2}\right), 26.9(t-\mathrm{Bu}), 19.2(\mathrm{C})$; HRMSESI $m / z$ calcd for $\mathrm{C}_{26} \mathrm{H}_{32} \mathrm{O}_{3} \mathrm{SiNa}[\mathrm{M}+\mathrm{Na}]^{+}: 443.2018$, found: 443.2018 .

(2S)-3-(tert-Butyldiphenylsilyloxy)-2-(methoxymethoxy)propanol (5b). Compound $5 \mathbf{b}$ was obtained from reduction of $(R)-\mathbf{4 b}$ using the general Method A of reduction. Oil (342 $\mathrm{mg}, 88 \%$ ); $R_{f} 0.33$ (EtOAc/cyclohexane, 3/7); $[\alpha]_{\mathrm{D}}{ }^{25}=-36.11$ (c 1.15, $\mathrm{CHCl}_{3}$ ); IR (neat) $v_{\max } 3452,3072$, 3052, 1473, 1428, 1112, 1106; ${ }^{1} \mathrm{H}$ NMR $\left(\mathrm{CDCl}_{3}\right) \delta$ 7.69-7.67 (m, 4H, H-Ar), 7.46-7.38 (m, 6H, $\mathrm{H}-\mathrm{Ar}$ ), 4.68 (s, 2H, O-CH $2-\mathrm{O}$ ), 3.83-3.66 (m, 5H, $\left.\mathrm{CH}_{2}-\mathrm{OH}, \mathrm{CH}-\mathrm{O}, \mathrm{CH}_{2}-\mathrm{O}\right), 3.37$ (s, 3H, $\mathrm{OCH}_{3}$ ), $2.67(\mathrm{dd}, 1 \mathrm{H}, J=8.0,5.0 \mathrm{~Hz}, \mathrm{OH}), 1.07(\mathrm{~s}, 9 \mathrm{H}, t-\mathrm{Bu}) ;{ }^{13} \mathrm{C} \mathrm{NMR}\left(\mathrm{CDCl}_{3}\right) \delta 135.6(\mathrm{C}-\mathrm{Ar}), 133.2$ (C-Ar), 129.8 (C-Ar), 127.8 (C-Ar), $96.7\left(\mathrm{O}-\mathrm{CH}_{2}-\mathrm{O}\right), 80.2(\mathrm{CH}-\mathrm{O}), 63.8\left(\mathrm{CH}_{2}-\mathrm{O}\right), 63.4\left(\mathrm{CH}_{2}-\right.$ $\mathrm{OH}), 55.6\left(\mathrm{O}-\mathrm{CH}_{3}\right), 26.8\left(t\right.$-Bu), $19.2(\mathrm{C})$; HRMS-ESI $m / z$ calcd for $\mathrm{C}_{21} \mathrm{H}_{30} \mathrm{O}_{4} \mathrm{SiNa}[\mathrm{M}+\mathrm{Na}]^{+}$: 397.1811, found: 397.1794 .

(2S)-2-(tert-Butyldimethylsilyloxy)-3-(tert-butyldiphenylsilyloxy)propanol (5c). Compound 5c was obtained from reduction of $(R)-4 \mathrm{c}$ using the general Method A of reduction. Oil $(0.217 \mathrm{~g}$, $47 \%) ; R_{f} 0.62($ EtOAc/cyclohexane, $1 / 9) ;[\alpha]_{\mathrm{D}}{ }^{25}=-15.86\left(\mathrm{c} 1.60, \mathrm{CHCl}_{3}\right)$; IR (neat) $v_{\max } 3450$, 3082, 3058, 1473, 1428, 1113, 1106; ${ }^{1} \mathrm{H} \mathrm{NMR}\left(\mathrm{CDCl}_{3}\right) \delta$ 7.69-7.65 (m, 4H, H-Ar), 7.46-7.36 (m, 6H, H-Ar), 3.78 (m, 1H, CH-O), 3.74-3.68 (m, 2H, $\left.\mathrm{CH}_{2}-\mathrm{O}\right), 3.64$ (m, 1H, $\left.\mathrm{CH}_{2}-\mathrm{I}\right), 3.59$ (dd, 1H, $J$ $\left.=10.5,5.0 \mathrm{~Hz}, \mathrm{CH}_{2}-\mathrm{I}\right), 1.05(\mathrm{~s}, 9 \mathrm{H}, t-\mathrm{Bu}), 0.84(\mathrm{~s}, 9 \mathrm{H}, t-\mathrm{Bu}), 0.01\left(\mathrm{~s}, 3 \mathrm{H}, \mathrm{CH}_{3}\right),-0.06(\mathrm{~s}, 3 \mathrm{H}$, $\left.\mathrm{CH}_{3}\right) ;{ }^{13} \mathrm{C} \mathrm{NMR}\left(\mathrm{CDCl}_{3}\right) \delta 135.6$ (C-Ar), 133.3 (C-Ar), 133.2 (C-Ar), 129.8 (C-Ar), 127.8 (C- 
Ar), 72.5 (CH-O), $65.1\left(\mathrm{CH}_{2}-\mathrm{O}\right), 64.7\left(\mathrm{CH}_{2}-\mathrm{OH}\right), 26.8(t$-Bu), $25.7(t-\mathrm{Bu}), 19.1(\mathrm{C}), 18.0(\mathrm{C})$, $5.0\left(\mathrm{CH}_{3}\right)$; HRMS-ESI $m / z$ calcd for $\mathrm{C}_{25} \mathrm{H}_{40} \mathrm{O}_{3} \mathrm{Si}_{2} \mathrm{Na}[\mathrm{M}+\mathrm{Na}]^{+}: 467.2414$, found: 467.2433.

Methyl (2R)-2-[(tert-butoxycarbonyl)amino]-3-hydroxypropanoate (6). To a stirred solution of D-serine methyl ester hydrochloride $(2.870 \mathrm{~g}, 18.40 \mathrm{mmol})$ in anhydrous tetrahydrofuran (60 $\mathrm{mL}$ ) was added, at $0{ }^{\circ} \mathrm{C}$ under argon atmosphere, triethylamine $(5.60 \mathrm{~mL}, 39.60 \mathrm{mmol})$ followed $15 \mathrm{~min}$ later by di-tert-butyl dicarbonate $(4.02 \mathrm{~g}, 18.40 \mathrm{mmol})$ in anhydrous tetrahydrofuran (30 $\mathrm{mL})$. The reaction mixture was stirred $12 \mathrm{~h}$ at $20{ }^{\circ} \mathrm{C}$ and then heated at $50{ }^{\circ} \mathrm{C}$ for $2 \mathrm{~h}$. After concentration, the residue was dissolved by addition of diethyl ether $(60 \mathrm{~mL})$ and water $(60 \mathrm{~mL})$. The aqueous layer was extracted. The organic layer was successively washed by a 3\% aqueous hydrochloric acid solution, a 5\% aqueous sodium bicarbonate solution and then by brine before being dried $\left(\mathrm{MgSO}_{4}\right)$. The solvent was then evaporated under vacuo to give $\mathbf{6}$ as a pale yellow oil (3.02 g, 75\%); $R_{f} 0.58$ (EtOAc/cyclohexane 7/3); $[\alpha]_{\mathrm{D}}^{25}=+17.8(\mathrm{c} 7.5, \mathrm{MeOH})$ (lit. for the $(S)$ isomer $^{11}[\alpha]_{\mathrm{D}}{ }^{25}=-17.5$ (c 5.0, MeOH)); IR (neat) $v_{\max } 3400,1749,1692 ;{ }^{1} \mathrm{H}$ NMR $\left(\mathrm{CDCl}_{3}\right) \delta$ 5.46 (bs, 1H, NH), 4.40 (bs, 1H, CH), 3.95 (m, 2H, $\left.\mathrm{CH}_{2}-\mathrm{O}\right), 3.79$ (s, 3H, OMe), 2.32 (bs, 1H, $\mathrm{OH}), 1.45(\mathrm{~s}, 9 \mathrm{H}, t-\mathrm{Bu}) ;{ }^{13} \mathrm{C} \mathrm{NMR}\left(\mathrm{CDCl}_{3}\right) \delta 171.4\left(\mathrm{CO}_{2} \mathrm{Me}\right), 155.7\left(\mathrm{CO}_{2} t-\mathrm{Bu}\right), 80.1(\mathrm{C}), 63.1$ $\left(\mathrm{CH}_{2} \mathrm{O}\right), 55.6(\mathrm{CH}), 52.5\left(\mathrm{OCH}_{3}\right), 28.2(t-\mathrm{Bu})$.

Methyl (2R)-2-(benzylamino)-3-hydroxypropanoate (7). To a stirred suspension of D-serine methyl ester hydrochloride $(3.90 \mathrm{~g}, 25.00 \mathrm{mmol})$ in methanol $(25 \mathrm{~mL})$ at $0{ }^{\circ} \mathrm{C}$ and under argon atmosphere, was added triethylamine $(3.5 \mathrm{~mL}, 25.0 \mathrm{mmol})$ followed after 15 min by benzaldehyde $(2.60 \mathrm{~mL}, 25.00 \mathrm{mmol})$. The reaction mixture was stirred $2 \mathrm{~h}$ at $20{ }^{\circ} \mathrm{C}$ then cold at $0{ }^{\circ} \mathrm{C}$. Sodium borohydride was slowly and portion wises added $(1.90 \mathrm{~g}, 50.00 \mathrm{mmol})$ followed $30 \mathrm{~min}$. later by a $4 \mathrm{M}$ hydrochloric acid solution $(50 \mathrm{~mL})$ and diethyl ether $(125 \mathrm{~mL})$. The organic layer was extracted and washed with a 4M hydrochloric acid solution $(50 \mathrm{~mL})$. The aqueous layers were then combined, neutralized with solid sodium bicarbonate and extracted with diethyl ether. The organic layer thus obtained was dried $\left(\mathrm{MgSO}_{4}\right)$ and the solvent was evaporated under vacuo to give $(R)-7(5.2 \mathrm{~g}, 99 \%)$ as a yellow oil. $R_{f} 0.60$ (EtOAc); $[\alpha]_{\mathrm{D}}{ }^{25}=+$ 36.6 (c 6.2, $\mathrm{CHCl}_{3}$ ); IR (neat) $v_{\max } 3325,1737 ;{ }^{1} \mathrm{H} \mathrm{NMR}\left(\mathrm{CDCl}_{3}\right) \delta 7.42-7.25$ (m, 5H, H-Ar), $3.87\left(\mathrm{dd}, J=13.0,4.5 \mathrm{~Hz}, 1 \mathrm{H}, \mathrm{CH}_{2} \mathrm{Ph}\right), 3.77$ (dd, $\left.J=11.0,4.5 \mathrm{~Hz}, 1 \mathrm{H}, \mathrm{CH}_{2}-\mathrm{OH}\right), 3.74(\mathrm{~s}, 3 \mathrm{H}$, $\left.\mathrm{O}-\mathrm{CH}_{3}\right), 3.73\left(\mathrm{~m}, 1 \mathrm{H}, \mathrm{CH}_{2} \mathrm{Ph}\right), 3.62$ (dd, $\left.J=11.0,6.5 \mathrm{~Hz}, 1 \mathrm{H}, \mathrm{CH}_{2}-\mathrm{OH}\right), 3.44$ (m, 1H, CH-N), 2.35 (bs, 2H, OH, NH); ${ }^{13} \mathrm{C}$ NMR $\left(\mathrm{CDCl}_{3}\right) \delta 173.4\left(\mathrm{CO}_{2} \mathrm{Me}\right), 139.2$ (C-Ar), 128.5 (C-Ar), 128.2 (C-Ar), 127.3 (C-Ar), $62.4\left(\mathrm{CH}_{2}-\mathrm{O}\right), 61.8(\mathrm{CH}-\mathrm{N}), 52.2\left(\mathrm{CH}_{2} \mathrm{Ph}\right), 52.0\left(\mathrm{OCH}_{3}\right) ; \mathrm{HRMS}-\mathrm{ESI} \mathrm{m} / z$ calcd for $\mathrm{C}_{11} \mathrm{H}_{16} \mathrm{NO}_{3}[\mathrm{M}+\mathrm{H}]^{+}: 210.1130$, found: 210.1136 .

Methyl (2R)-2-[benzyl(tert-butoxycarbonyl)amino]-3-hydroxypropanoate (8). To a stirred solution of $(R)-7(4.00 \mathrm{~g}, 19.10 \mathrm{mmol})$ in anhydrous diethyl ether $(6 \mathrm{~mL})$ was added under argon di-tert-butyl dicarbonate $(4.00 \mathrm{~g}, 18.10 \mathrm{mmol})$. The reaction mixture was stirred $12 \mathrm{~h}$ at $20{ }^{\circ} \mathrm{C}$. The solution was then successively washed with a $0.1 \mathrm{M}$ hydrochloric acid solution, a saturated aqueous sodium bicarbonate solution and brine. The organic layer was dried $\left(\mathrm{MgSO}_{4}\right)$ and the solvent was evaporated under reduced pressure. The residue was purified by flash column chromatography (ethyl acetate/cyclohexane, 2:3) affording 8 as a pale yellow oil (4.00 g, 71\%). $R_{f} 0.70$ (EtOAc/cyclohexane 2/3); $[\alpha]_{\mathrm{D}}{ }^{25}=+28.7\left(\mathrm{c} 2.4, \mathrm{CHCl}_{3}\right) ;{ }^{13} \mathrm{C} \mathrm{NMR}\left(\mathrm{CDCl}_{3}\right) \delta 173.4$ 
$\left(\mathrm{CO}_{2} \mathrm{Me}\right), 155.3\left(\mathrm{CO}_{2} t-\mathrm{Bu}\right), 137.9$ (C-Ar), 137.5 (C-Ar), 128.6 (C-Ar), 128.3 (C-Ar), 127.7 (C$\mathrm{Ar}), 127.5(\mathrm{C}-\mathrm{Ar}), 81.3(\mathrm{C}), 61.8\left(\mathrm{CH}_{2}-\mathrm{O}\right), 61.0(\mathrm{CH}-\mathrm{N}), 52.2\left(\mathrm{CH}_{2} \mathrm{Ph}\right), 52.0\left(\mathrm{OCH}_{3}\right), 28.2(t-$ $\mathrm{Bu})$.

Methyl (2R)-2-[benzyl(tert-butoxycarbonyl)amino]-3-(tert-butyldimethylsilyloxy)propaneate (9a). To a stirred solution of $(R)-8(4.00 \mathrm{~g}, 12.90 \mathrm{mmol})$ and imidazole $(3.50 \mathrm{~g}, 51.60 \mathrm{mmol})$ in anhydrous dichloromethane $(60 \mathrm{~mL})$ was added dropwise under argon atmosphere a solution of tert-butyldimethylsilyl chloride $(2.90 \mathrm{~g}, 19.40 \mathrm{mmol})$ in anhydrous dichloromethane $(60 \mathrm{~mL})$. The reaction was stirred for $3 \mathrm{~h}$ at $20^{\circ} \mathrm{C}$ before being diluted with diethyl ether. The organic layer was washed with water and dried $\left(\mathrm{MgSO}_{4}\right)$. The solvent was evaporated and the residue was purified by flash column chromatography (ethyl acetate/cyclohexane, 1:4) furnishing 9a as a colourless oil $(4.40 \mathrm{~g}, 80 \%)$ which appeared at room temperature in the NMR spectra as a mixture of two rotamers $a$ and $b ; R_{f} 0.28$ (EtOAc/cyclohexane 1/4); $[\alpha]_{\mathrm{D}}{ }^{25}=+32.2$ (c 2.9, $\left.\mathrm{CHCl}_{3}\right) ;{ }^{1} \mathrm{H}$ NMR $\left(\mathrm{CDCl}_{3}\right) \delta 7.35-7.15(\mathrm{~m}, 5 \mathrm{H}, \mathrm{H}-\mathrm{Ar}, a$ and $b), 4.95\left(\mathrm{~d}, J=15.0 \mathrm{~Hz}, 1 \mathrm{H}, \mathrm{CH}_{2} \mathrm{Ph}\right.$, a), $4.72\left(\mathrm{~d}, J=16.0 \mathrm{~Hz}, 1 \mathrm{H}, \mathrm{CH}_{2} \mathrm{Ph}, b\right), 4.50(\mathrm{~m}, 1 \mathrm{H}, \mathrm{CH}, b), 4.39\left(\mathrm{~d}, J=16.0 \mathrm{~Hz}, 1 \mathrm{H}, \mathrm{CH}_{2} \mathrm{Ph}\right.$, b), $4.28\left(\mathrm{~d}, J=15.0 \mathrm{~Hz}, 1 \mathrm{H}, \mathrm{CH}_{2} \mathrm{Ph}, a\right), 4.10-3.90\left(\mathrm{~m}, 3 \mathrm{H}, \mathrm{CH}_{2}-\mathrm{O}, a\right.$ and $\left.b, \mathrm{CH}, a\right), 3.63(\mathrm{~s}, 3 \mathrm{H}$, $\left.\mathrm{O}-\mathrm{CH}_{3}, b\right), 3.59\left(\mathrm{~s}, 3 \mathrm{H}, \mathrm{O}-\mathrm{CH}_{3}, a\right), 1.41(\mathrm{~s}, 9 \mathrm{H}, t-\mathrm{Bu}, a), 1.33(\mathrm{~s}, 9 \mathrm{H}, t-\mathrm{Bu}, b), 0.88(\mathrm{~s}, 9 \mathrm{H}, t-\mathrm{Bu}$, a), $0.82(\mathrm{~s}, 9 \mathrm{H}, t-\mathrm{Bu}, b), 0.00\left(\mathrm{~s}, 6 \mathrm{H}, \mathrm{CH}_{3}, a\right),-0.02\left(\mathrm{~s}, 3 \mathrm{H}, \mathrm{CH}_{3}, b\right),-0.08\left(\mathrm{~s}, 3 \mathrm{H}, \mathrm{CH}_{3}, b\right) ;{ }^{13} \mathrm{C}$ NMR $\left(\mathrm{CDCl}_{3}\right) \delta 170.4\left(\mathrm{CO}_{2} \mathrm{Me}, a\right.$ and $\left.b\right), 155.7\left(\mathrm{CO}_{2} t \mathrm{Bu}, b\right), 155.1\left(\mathrm{CO}_{2} t \mathrm{Bu}, a\right), 139.3(\mathrm{C}-\mathrm{Ar}$, b), 138.1 (C-Ar, $a$ ), 128.2 (C-Ar, $a$ and $b$ ), 128.0 (C-Ar, $b$ ), 127.0 (C-Ar, $a), 126.6$ (C-Ar, $a$ and b), $80.7(\mathrm{C}, a), 80.4(\mathrm{C}, b), 62.4\left(\mathrm{CH}_{2}-\mathrm{O}, a\right), 62.0\left(\mathrm{CH}_{2}-\mathrm{O}, b\right), 61.3(\mathrm{CH}-\mathrm{N}, a), 61.0(\mathrm{CH}-\mathrm{N}, b)$, $51.9\left(\mathrm{CH}_{2} \mathrm{Ph}, a\right), 51.8\left(\mathrm{O}-\mathrm{CH}_{3}, b\right), 51.7\left(\mathrm{O}-\mathrm{CH}_{3}, a\right), 51.3\left(\mathrm{CH}_{2} \mathrm{Ph}, b\right), 28.3(t-\mathrm{Bu}, a), 28.2(t-\mathrm{Bu}$, $b), 25.8(t$-Bu, $a$ and $b), 18.1(\mathrm{C}, a$ and $b),-3.6\left(\mathrm{CH}_{3}, a\right),-5.6\left(\mathrm{CH}_{3}, b\right)$. HRMS-ESI $m / z$ calcd for $\mathrm{C}_{22} \mathrm{H}_{37} \mathrm{NO}_{5} \mathrm{SiNa}[\mathrm{M}+\mathrm{Na}]^{+}: 446.2339$, found: 446.2359 .

Methyl (2R)-2-[(tert-butoxycarbonyl)amino]-3-(tert-butyldiphenylsilyloxy)propanoate (9b). To a stirred solution of $(R)-6(0.800 \mathrm{~g}, 3.65 \mathrm{mmol})$ in anhydrous dichloromethane $(10 \mathrm{~mL})$ at 0 ${ }^{\circ} \mathrm{C}$ and under argon atmosphere was added tert-butyldiphenylsilyl chloride $(1.90 \mathrm{~mL}, 7.30$ mmol) followed slowly by a solution of imidazole $(486 \mathrm{mg}, 7.30 \mathrm{mmol}$ ) in anhydrous dichloromethane $(2 \mathrm{~mL})$. The reaction mixture was then stirred at $20^{\circ} \mathrm{C}$ for $12 \mathrm{~h}$ and quenched with water $(10 \mathrm{~mL})$. The organic layer was washed twice with water. After drying $\left(\mathrm{MgSO}_{4}\right)$, the solvent was evaporated in vacuo and the residue was purified by flash column chromatography (diethyl ether/pentane, 1:9) furnishing $9 \mathbf{b}$ as a yellow oil (1.64 g, 98\%). $R_{f} 0.48$ (EtOAc/cyclohexane 3/7); $[\alpha]_{\mathrm{D}}{ }^{25}=-15.5\left(\mathrm{c} 4.5, \mathrm{CHCl}_{3}\right)\left(\right.$ for $(S)-9 \mathbf{b}$ lit $^{12 \mathrm{a}}[\alpha]_{\mathrm{D}}{ }^{25}=+14.2(\mathrm{c} 1.0$, $\mathrm{CHCl}_{3}$ ) and $\operatorname{lit}^{12 \mathrm{~b}}[\alpha]_{\mathrm{D}}{ }^{25}=+14.3\left(\mathrm{c} 1.0, \mathrm{CHCl}_{3}\right)$; IR (neat) $v_{\max } 3447,1750,1719 ;{ }^{1} \mathrm{H} \mathrm{NMR}$ $\left(\mathrm{CDCl}_{3}\right) \delta 7.61(\mathrm{~m}, 4 \mathrm{H}, \mathrm{H}-\mathrm{Ar}), 7.40(\mathrm{~m}, 6 \mathrm{H}, \mathrm{H}-\mathrm{Ar}), 5.42(\mathrm{~d}, J=9.0 \mathrm{~Hz}, 1 \mathrm{H}, \mathrm{NH}), 4.40(\mathrm{dt}, J=$ 9.0, 3.0 Hz, 1H, CH), 4.07 (dd, $J=10.0,3.0 \mathrm{~Hz}, 1 \mathrm{H}, \mathrm{CH}_{2}-\mathrm{O}$ ), 3.89 (dd, $J=10.0,3.0 \mathrm{~Hz}, 1 \mathrm{H}$, $\mathrm{CH}_{2}-\mathrm{O}$ ), $3.74\left(\mathrm{~s}, 3 \mathrm{H}, \mathrm{OCH}_{3}\right), 1.47(\mathrm{~s}, 9 \mathrm{H}, t-\mathrm{Bu}), 1.03(\mathrm{~s}, 9 \mathrm{H}, t-\mathrm{Bu}) ;{ }^{13} \mathrm{C} \mathrm{NMR}\left(\mathrm{CDCl}_{3}\right) \delta 171.2$ $\left(\mathrm{CO}_{2} \mathrm{Me}\right), 155.3\left(\mathrm{CO}_{2} t \mathrm{Bu}\right), 135.5$ (C-Ar), 135.4 (C-Ar), 132.9 (C-Ar), 132.7 (C-Ar), $129.8(\mathrm{C}-$ Ar), $127.7(\mathrm{C}-\mathrm{Ar}), 79.9(\mathrm{C}), 64.6\left(\mathrm{CH}_{2}-\mathrm{O}\right), 55.5(\mathrm{CH}), 52.2\left(\mathrm{OCH}_{3}\right), 28.3(t-\mathrm{Bu}), 26.7(t-\mathrm{Bu})$, 19.2 (C); MS (ESI) $m / z 481\left([\mathrm{M}+\mathrm{Na}+1]^{+}, 30\right), 480\left([\mathrm{M}+\mathrm{Na}]^{+}, 100\right), 424(10)$. 
Methyl [(4R)-3-(tert-butoxycarbonyl)-2,2-dimethyl-1,3-oxazolidin-4-yl]carboxylate (9c). Compound 9c was prepared from $(R)-6$ following the general procedure of preparation of oxazolidines and appeared in the NMR spectra at room temperature, as a mixture of two rotamers $a$ and $b$. Pale yellow oil $(0.746 \mathrm{~g}, 83 \%) ; R_{f} 0.67($ EtOAc/cyclohexane $2 / 3) ;[\alpha]_{\mathrm{D}}{ }^{25}=+$ $53.6\left(\mathrm{c} 2.02, \mathrm{CHCl}_{3}\right)\left(\mathrm{lit}^{13 \mathrm{~b}}[\alpha]_{\mathrm{D}}{ }^{20}=+49.8\left(\mathrm{c} 1.04, \mathrm{CHCl}_{3}\right)\right)$; IR (neat) $v_{\max } 1758,1708 ;{ }^{1} \mathrm{H} \mathrm{NMR}$ $\left(\mathrm{CDCl}_{3}\right) \delta 4.48(\mathrm{dd}, J=7.0,2.5 \mathrm{~Hz}, 1 \mathrm{H}, \mathrm{CH}, a), 4.37(\mathrm{dd}, J=7.0,3.0 \mathrm{~Hz}, 1 \mathrm{H}, \mathrm{CH}, b), 4.15(\mathrm{dd}$, $\left.J=9.0,7.0 \mathrm{~Hz}, 1 \mathrm{H}, \mathrm{CH}_{2}-\mathrm{O}, a\right), 4.12\left(\mathrm{dd}, J=9.0,7.0 \mathrm{~Hz}, 1 \mathrm{H}, \mathrm{CH}_{2}-\mathrm{O}, b\right), 4.05$ (dd, $J=9.0,2.5$ $\left.\mathrm{Hz}, 1 \mathrm{H}, \mathrm{CH}_{2}-\mathrm{O}, a\right), 4.02\left(\mathrm{dd}, J=9.0,3.0 \mathrm{~Hz}, 1 \mathrm{H}, \mathrm{CH}_{2}-\mathrm{O}, b\right), 3.75\left(\mathrm{~s}, 3 \mathrm{H}, \mathrm{O}-\mathrm{CH}_{3}, a\right.$ and $\left.b\right), 1.67$ $\left(\mathrm{s}, 3 \mathrm{H}, \mathrm{CH}_{3}, b\right), 1.63\left(\mathrm{~s}, 3 \mathrm{H}, \mathrm{CH}_{3}, a\right), 1.53\left(\mathrm{~s}, 3 \mathrm{H}, \mathrm{CH}_{3}, b\right), 1.49(\mathrm{~s}, 9 \mathrm{H}, t-\mathrm{Bu} a), 1.42\left(\mathrm{~s}, 3 \mathrm{H}, \mathrm{CH}_{3}\right.$, a), $1.41(\mathrm{~s}, 9 \mathrm{H}, t-\mathrm{Bu}, b) ;{ }^{13} \mathrm{C} \mathrm{NMR}\left(\mathrm{CDCl}_{3}\right) \delta 171.5\left(\mathrm{CO}_{2} \mathrm{Me}, b\right), 171.0\left(\mathrm{CO}_{2} \mathrm{Me}, a\right), 151.9$ $\left(\mathrm{CO}_{2} t \mathrm{Bu}, a\right), 151.0\left(\mathrm{CO}_{2} t \mathrm{Bu}, b\right), 94.8(\mathrm{C}, b), 94.2(\mathrm{C}, a), 80.6(\mathrm{C}, a), 80.0(\mathrm{C}, b), 66.0\left(\mathrm{CH}_{2}-\mathrm{O}\right.$, $b), 65.8\left(\mathrm{CH}_{2}-\mathrm{O}, a\right), 59.1(\mathrm{CH}, b), 59.0(\mathrm{CH}, a), 52.1\left(\mathrm{O}-\mathrm{CH}_{3}, a\right), 52.0\left(\mathrm{O}-\mathrm{CH}_{3}, b\right), 28.1(t-\mathrm{Bu}, a)$, $28.0(t-\mathrm{Bu}, b), 25.8\left(\mathrm{CH}_{3}, b\right), 25.0\left(\mathrm{CH}_{3}, b\right), 24.7\left(\mathrm{CH}_{3}, a\right), 24.2\left(\mathrm{CH}_{3}, a\right)$.

tert-Butyl $N$-benzyl- $N$-[(1S)-1-[(tert-butyldimethylsilyloxy)methyl]-2-hydroxyethyl]carbamate (10a). Compound 10a was obtained from reduction of $(R)-9$ a using the general Method $\mathrm{B}$ of reduction. Colourless oil $(0.588 \mathrm{~g}, 95 \%) ; R_{f} 0.49$ (EtOAc/cyclohexane $\left.3 / 7\right)$; $[\alpha]_{\mathrm{D}}{ }^{25}=+6.3$ (c 4.4, $\left.\mathrm{CHCl}_{3}\right) ;{ }^{1} \mathrm{H}$ NMR $\left(\mathrm{CDCl}_{3}\right) \delta 7.35-7.15(\mathrm{~m}, 5 \mathrm{H}, \mathrm{H}-\mathrm{Ar}), 4.70\left(\mathrm{~m}, 1 \mathrm{H}, \mathrm{CH}_{2} \mathrm{Ph}\right), 4.30(\mathrm{~m}, 1 \mathrm{H}$, $\left.\mathrm{CH}_{2} \mathrm{Ph}\right), 4.00-3.60\left(\mathrm{~m}, 5 \mathrm{H}, \mathrm{CH}_{2}-\mathrm{OH}, \mathrm{CH}_{2}-\mathrm{O}, \mathrm{CH}\right), 1.80$ (bs, $\left.1 \mathrm{H}, \mathrm{OH}\right), 1.41(\mathrm{~s}, 9 \mathrm{H}, t \mathrm{Bu}), 0.85$ (s, $\left.9 \mathrm{H}, \mathrm{CH}_{3}\right), 0.00\left(\mathrm{~s}, 6 \mathrm{H}, \mathrm{CH}_{3}\right) ;{ }^{13} \mathrm{C} \mathrm{NMR}\left(\mathrm{CDCl}_{3}\right) \delta 156.6\left(\mathrm{CO}_{2} t \mathrm{Bu}\right), 139.2(\mathrm{C}-\mathrm{Ar}), 128.5(\mathrm{C}-\mathrm{Ar})$, 128.2 (C-Ar), 127.1 (C-Ar), 80.5 (C), $63.3\left(\mathrm{CH}_{2}-\mathrm{O}\right), 62.0(\mathrm{CH}-\mathrm{N}), 61.9\left(\mathrm{CH}_{2}-\mathrm{OH}\right), 52.4$ $\left(\mathrm{CH}_{2} \mathrm{Ph}\right), 28.3(t \mathrm{Bu}), 25.7\left(\mathrm{CH}_{3}\right), 18.1\left(\mathrm{CH}_{3}\right),-5.6\left(\mathrm{CH}_{3}\right)$.

tert-Butyl $N$-[(1S)-1-[(tert-butyldiphenylsilyloxy)methyl]-2-hydroxyethyl]carbamate (10b). Compound 10b was obtained from reduction of $(R)-9 \mathbf{b}$ using the general Method A of reduction. White solid (0.442 g, 99\%); $m p$ 77-78 ${ }^{\circ} \mathrm{C}\left(\right.$ EtOAc); $R_{f} 0.60($ EtOAc/cyclohexane $2 / 3) ;[\alpha]_{\mathrm{D}}{ }^{25}=$ $4.7\left(\mathrm{c} 3.7, \mathrm{CHCl}_{3}\left(\mathrm{lit}^{14}[\alpha]_{\mathrm{D}}{ }^{25}=-4.1\left(\mathrm{c} 1.12, \mathrm{CHCl}_{3}\right)\right) ;{ }^{1} \mathrm{H} \mathrm{NMR}\left(\mathrm{CDCl}_{3}\right) \delta 7.65\right.$ (m, 4H, H-Ar), 7.41 (M, 6H, H-Ar), 5.09 (d, $J=5.0 \mathrm{~Hz}, 1 \mathrm{H}, \mathrm{NH}), 3.84-3.65$ (m, 5H, CH, $\left.\mathrm{CH}_{2}-\mathrm{O}, \mathrm{CH}_{2}-\mathrm{OH}\right)$, $2.53(\mathrm{bs}, 1 \mathrm{H}, \mathrm{OH}), 1.44(\mathrm{~s}, 9 \mathrm{H}, t-\mathrm{Bu}), 1.07(\mathrm{~s}, 9 \mathrm{H}, t-\mathrm{Bu}) ;{ }^{13} \mathrm{C} \mathrm{NMR}\left(\mathrm{CDCl}_{3}\right) \delta 156.1\left(\mathrm{CO}_{2} t \mathrm{Bu}\right)$, 135.5 (C-Ar), 132.8 (C-Ar), 129.9 (C-Ar), 127.8 (C-Ar), 79.6 (C), $64.1\left(\mathrm{CH}_{2}-\mathrm{O}\right), 63.6\left(\mathrm{CH}_{2-}\right.$ $\mathrm{OH}), 53.0(\mathrm{CH}-\mathrm{N}), 28.3\left(\mathrm{CH}_{3}\right), 26.8\left(\mathrm{CH}_{3}\right), 19.2(\mathrm{C})$.

tert-Butyl [(4S)-2,2-dimethyl-4-(hydroxymethyl)-1,3-oxazolidin-3-yl]carboxylate (10c). Compound 10c was obtained from reduction of $(R)-9 \mathrm{c}$ using the general Method $\mathrm{C}$ of reduction and was detected at room temperature as a mixture of two rotamers $a$ and $b$. Colourless oil $(0.420 \mathrm{~g}, 95 \%) ; R_{f} 0.30($ EtOAc/cyclohexane $2 / 3) ;[\alpha]_{\mathrm{D}}{ }^{25}=+24.5\left(\mathrm{c} 2.3, \mathrm{CHCl}_{3}\right)\left(\right.$ lit. $^{13 \mathrm{~b}}[\alpha]_{\mathrm{D}}{ }^{20}=$ $+23.6\left(\mathrm{c} 1.44, \mathrm{CHCl}_{3}\right)$ and lit. ${ }^{13 \mathrm{a}}[\alpha]_{\mathrm{D}}{ }^{20}=+17.9\left(\mathrm{c} 2.13, \mathrm{CHCl}_{3}\right)$ ); IR (neat) $v 3427,1697 ;{ }^{1} \mathrm{H}$ NMR $\left(\mathrm{CDCl}_{3}\right) \delta 4.02-3.88\left(\mathrm{~m}, 3 \mathrm{H}, \mathrm{CH}, \mathrm{CH}_{2}-\mathrm{O}\right), 3.77-3.54\left(\mathrm{~m}, 3 \mathrm{H}, \mathrm{CH}_{2}-\mathrm{OH}, \mathrm{OH}\right), 1.53(\mathrm{~s}, 3 \mathrm{H}$, $\left.\mathrm{CH}_{3}, a\right), 1.50\left(\mathrm{~s}, 3 \mathrm{H}, \mathrm{CH}_{3}, b\right), 1.44\left(\mathrm{~s}, 12 \mathrm{H}, \mathrm{CH}_{3}\right.$ and $t-\mathrm{Bu} a$ and $\left.b\right) ;{ }^{13} \mathrm{C} \mathrm{NMR}\left(\mathrm{CDCl}_{3}\right) \delta 153.9$ $\left(\mathrm{CO}_{2} t \mathrm{Bu}, a\right.$ and $\left.b\right), 93.9(\mathrm{C}, a$ and $b), 81.0(\mathrm{C}, a$ and $b), 65.2\left(\mathrm{CH}_{2}-\mathrm{O}, b\right), 64.7\left(\mathrm{CH}_{2}-\mathrm{O}, a\right), 64.7$ $\left(\mathrm{CH}_{2}-\mathrm{O}, b\right), 62.7\left(\mathrm{CH}_{2}-\mathrm{O}, a\right), 59.3(\mathrm{CH}, b), 58.3(\mathrm{CH}, a), 28.3(t-\mathrm{Bu}, a$ and $b), 27.1\left(\mathrm{CH}_{3}, b\right)$, $26.6\left(\mathrm{CH}_{3}, a\right), 24.4\left(\mathrm{CH}_{3}, b\right), 22.9\left(\mathrm{CH}_{3}, a\right)$. 
tert-Butyl $\quad N$-[(1R)-1-[(tert-butyldiphenylsilyloxy)methyl]-2-iodoethyl]carbamate (11b). Compound 11b was prepared from alcohol $(S)$-10b in toluene following the general procedure for the preparation of iodides. Yellow oil $(0.424 \mathrm{~g}, 77 \%) ; R_{f} 0.71$ (EtOAc/cyclohexane 1/4); $[\alpha]_{\mathrm{D}}{ }^{25}=+8.79\left(\mathrm{c} 7.3, \mathrm{CHCl}_{3}\right) ; \mathrm{IR}$ (neat) $v_{\max } 3431,1717 ;{ }^{1} \mathrm{H} \mathrm{NMR}\left(\mathrm{CDCl}_{3}\right) \delta 7.66(\mathrm{~d}, J=7.0$ $\mathrm{Hz}, 4 \mathrm{H}, \mathrm{H}-\mathrm{Ar}$ ), 7.45 (t, $J=8.0 \mathrm{~Hz}, 2 \mathrm{H}, \mathrm{H}-\mathrm{Ar}), 7.40$ (t, $J=8.0 \mathrm{~Hz}, 4 \mathrm{H}, \mathrm{H}-\mathrm{Ar}), 4.81$ (d, $J=8.0 \mathrm{~Hz}$, $1 \mathrm{H}, \mathrm{NH}), 3.84\left(\mathrm{dd}, J=10.0,3.0 \mathrm{~Hz}, 1 \mathrm{H}, \mathrm{CH}_{2}-\mathrm{O}\right), 3.70(\mathrm{~m}, 1 \mathrm{H}, \mathrm{CH}), 3.62(\mathrm{dd}, J=10.0,5.5 \mathrm{~Hz}$, $1 \mathrm{H}, \mathrm{CH}_{2}-\mathrm{O}$ ), 3.49 (dd, $\left.J=15.5,7.0 \mathrm{~Hz}, 1 \mathrm{H}, \mathrm{CH}_{2}-\mathrm{I}\right), 3.43$ (dd, $\left.J=15.5,9.0 \mathrm{~Hz}, 1 \mathrm{H}, \mathrm{CH}_{2}-\mathrm{I}\right), 1.45$ $(\mathrm{s}, 9 \mathrm{H}, t-\mathrm{Bu}), 1.08(\mathrm{~s}, 9 \mathrm{H}, t-\mathrm{Bu}) ;{ }^{13} \mathrm{C} \mathrm{NMR}\left(\mathrm{CDCl}_{3}\right) \delta 154.9\left(\mathrm{CO}_{2} t-\mathrm{Bu}\right), 135.6(\mathrm{C}-\mathrm{Ar}), 132.9(\mathrm{C}-$ Ar), 132.8 (C-Ar), 129.9 (C-Ar), 127.8 (C-Ar), 79.8 (C), $64.5\left(\mathrm{CH}_{2}-\mathrm{O}\right), 51.8(\mathrm{CH}), 28.3(t$-Bu), $26.8(t \mathrm{Bu}), 19.3(\mathrm{C}), 8.7\left(\mathrm{CH}_{2}-\mathrm{I}\right)$; HRMS-ESI $\mathrm{m} / \mathrm{z}$ calcd for $\mathrm{C}_{24} \mathrm{H}_{34} \mathrm{INO}_{3} \mathrm{SiNa}[\mathrm{M}+\mathrm{Na}]^{+}$: 562.1250, found: 562.1241 .

tert-Butyl [(4R)-2,2-dimethyl-4-(iodomethyl)-1,3-oxazolidin-3-yl]carboxylate

(11c). Compound 11c was prepared from alcohol $(S)-10 \mathrm{c}$ in toluene following the general procedure for the preparation of iodides and appeared in the NMR spectra at ambient temperature as a mixture of two rotamers $a$ and $b$. Colourless liquid $(0.257 \mathrm{mg}, 74 \%) ; R_{f} 0.46$ (EtOAc/cyclohexane 1/9); $[\alpha]_{\mathrm{D}}^{25}=-5.5\left(\mathrm{c} 1.86, \mathrm{CHCl}_{3}\right)$; IR (neat) $v_{\max } 1698 ;{ }^{1} \mathrm{H} \mathrm{NMR}\left(\mathrm{CDCl}_{3}\right) \delta 4.18(\mathrm{~m}, 1 \mathrm{H}, \mathrm{CH}, a)$, $4.08(\mathrm{~m}, 1 \mathrm{H}, \mathrm{CH}, b), 3.99\left(\mathrm{~m}, 2 \mathrm{H}, \mathrm{CH}_{2}-\mathrm{O}, a\right.$ and $\left.b\right), 3.50\left(\mathrm{dd}, J=9.5,2.5 \mathrm{~Hz}, 1 \mathrm{H}, \mathrm{CH}_{2}-\mathrm{I}, b\right), 3.36$ (bd, $\left.J=9.0 \mathrm{~Hz}, 1 \mathrm{H}, \mathrm{CH}_{2}-\mathrm{I}, a\right), 3.14$ (t, $\left.J=9.0 \mathrm{~Hz}, 1 \mathrm{H}, \mathrm{CH}_{2}-\mathrm{I}, a\right), 3.12$ (t, $J=9.5 \mathrm{~Hz}, 1 \mathrm{H}, \mathrm{CH}_{2}-\mathrm{I}$, b), $1.61\left(\mathrm{~s}, 3 \mathrm{H}, \mathrm{CH}_{3}, b\right), 1.56\left(\mathrm{~s}, 3 \mathrm{H}, \mathrm{CH}_{3}, a\right), 1.47(\mathrm{~s}, 9 \mathrm{H}, t-\mathrm{Bu}, a), 1.46(\mathrm{~s}, 9 \mathrm{H}, t-\mathrm{Bu}, b), 1.46(\mathrm{~s}$, $\left.3 \mathrm{H}, \mathrm{CH}_{3}, a\right), 1.43\left(\mathrm{~s}, 3 \mathrm{H}, \mathrm{CH}_{3}, b\right) ;{ }^{13} \mathrm{C} \mathrm{NMR}\left(\mathrm{CDCl}_{3}\right) \delta 152.0\left(\mathrm{CO}_{2} t \mathrm{Bu}, a\right), 151.3\left(\mathrm{CO}_{2} t \mathrm{Bu}, b\right)$, $94.8(\mathrm{C}, b), 94.3(\mathrm{C}, a), 80.6(\mathrm{C}, a), 80.3(\mathrm{C}, b), 67.2\left(\mathrm{CH}_{2}-\mathrm{O}, a\right.$ and $\left.b\right), 59.0(\mathrm{CH}, b), 58.9(\mathrm{CH}$, a), $28.4(t-\mathrm{Bu}, b), 28.3(t-\mathrm{Bu}, a), 27.8\left(\mathrm{CH}_{3}, a\right), 27.1\left(\mathrm{CH}_{3}, b\right), 24.3\left(\mathrm{CH}_{3}, a\right), 23.0\left(\mathrm{CH}_{3}, b\right), 6.8$ $\left(\mathrm{CH}_{2}-\mathrm{I}, b\right), 6.7\left(\mathrm{CH}_{2}-\mathrm{I}, a\right)$; HRMS-ESI $m / z$ calcd for $\mathrm{C}_{11} \mathrm{H}_{20} \mathrm{INO}_{3} \mathrm{Na}[\mathrm{M}+\mathrm{Na}]^{+}: 364.0386$, found: 364.0385 .

(2S)-2-[(tert-Butoxycarbonyl)amino]-4-methoxy-4-oxobutanoic acid (12). To a stirred solution of L-aspartic acid methyl ester hydrochloride $(1.00 \mathrm{~g}, 5.4 \mathrm{mmol})$ in dioxane $(20 \mathrm{~mL})$ was added at $0{ }^{\circ} \mathrm{C}$ a $0.5 \mathrm{M}$ aqueous sodium carbonate solution $(22 \mathrm{~mL})$ followed by di-tert-butyl dicarbonate $(1.30 \mathrm{~g}, 6.0 \mathrm{mmol})$. The reaction mixture was stirred $12 \mathrm{~h}$ at $20{ }^{\circ} \mathrm{C}$ before being concentrated. The resulting solution was acidified at $0{ }^{\circ} \mathrm{C}(\mathrm{pH}=3.0)$ by adjunction of citric acid and extracted with ethyl acetate $(3 \times 20 \mathrm{~mL})$. The combined organic layers were washed by water and dried $\left(\mathrm{MgSO}_{4}\right)$. The solvent was evaporated under vacuo to give a residue which was purified by flash column chromatography (ethanol/dichloromethane, 1/5) leading to $(S)$-12 as a pale yellow oil $(1.19 \mathrm{~g}, 89 \%) . R_{f} 0.64\left(\mathrm{EtOH} / \mathrm{CH}_{2} \mathrm{Cl}_{2} 1 / 5\right) ;[\alpha]_{\mathrm{D}}{ }^{25}=+23.5\left(\mathrm{c} 0.26, \mathrm{CHCl}_{3}\right)$; IR (neat) $v 3300,1750-1680 ;{ }^{1} \mathrm{H}$ NMR $\left(\mathrm{CDCl}_{3}\right) \delta 9.88\left(\mathrm{bs}, 1 \mathrm{H}, \mathrm{CO}_{2} \mathrm{H}\right), 5.61(\mathrm{~d}, J=8.0 \mathrm{~Hz}, 1 \mathrm{H}$, $\mathrm{NH}), 4.58(\mathrm{dt}, J=8.0,4.0 \mathrm{~Hz}, 1 \mathrm{H}, \mathrm{CH}), 3.68\left(\mathrm{~s}, 3 \mathrm{H}, \mathrm{O}-\mathrm{CH}_{3}\right), 2.99$ (dd, $J=17.0,4.0 \mathrm{~Hz}, 1 \mathrm{H}$, $\left.\mathrm{CH}_{2}\right), 2.83\left(\mathrm{dd}, J=17.0,4.5 \mathrm{~Hz}, 1 \mathrm{H}, \mathrm{CH}_{2}\right), 1.42(\mathrm{~s}, 9 \mathrm{H}, t-\mathrm{Bu}) ;{ }^{13} \mathrm{C} \mathrm{NMR}\left(\mathrm{CDCl}_{3}\right) \delta 175.4$ $\left(\mathrm{CO}_{2} \mathrm{H}\right), 171.6\left(\mathrm{CO}_{2} \mathrm{Me}\right), 155.6\left(\mathrm{CO}_{2} t \mathrm{Bu}\right), 80.4(\mathrm{C}), 52.0\left(\mathrm{O}-\mathrm{CH}_{3}\right), 49.8(\mathrm{CH}), 36.4\left(\mathrm{CH}_{2}\right), 28.2$ $\left(t\right.$-Bu); HRMS-ESI $m / z$ calcd for $\mathrm{C}_{10} \mathrm{H}_{17} \mathrm{NO}_{6} \mathrm{Na}[\mathrm{M}+\mathrm{Na}]^{+}: 270.0954$, found:270.0963.

Methyl (3S)-3-[(tert-butoxycarbonyl)amino]-4-hydroxybutanoate (13). To a stirred solution of $(S)-12(1.500 \mathrm{~g}, 6.4 \mathrm{mmol})$ and $N$-hydroxysuccinimide $(0.838 \mathrm{~g}, 7.7 \mathrm{mmol})$ in anhydrous 
dichloromethane $(20 \mathrm{~mL})$, was added, at $20{ }^{\circ} \mathrm{C}$ and under argon atmosphere, $N, N^{\prime}-$ dicyclohexylcarbodiimide $(1.504 \mathrm{~g}, 7.7 \mathrm{mmol})$. The reaction mixture was stirred for $12 \mathrm{~h}$ at 20 ${ }^{\circ} \mathrm{C}$ and anhydrous $\mathrm{MgSO}_{4}(1.0 \mathrm{~g})$ was added. After $15 \mathrm{~min}$, the solution was filtrated and the solvent was evaporated. The residue was dissolved by a $3 / 1$ (v/v) mixture of tetrahydrofuran/ethanol $(30 \mathrm{~mL})$ and the resulting solution was placed at $0{ }^{\circ} \mathrm{C}$. Sodium borohydride $(0.229 \mathrm{~g}, 6.4 \mathrm{mmol})$ was portionwise added and the stirring was pursued at this temperature $30 \mathrm{~min}$ after complete addition. The reaction mixture was diluted by ethyl acetate and filtered. The organic layer was dried $\left(\mathrm{MgSO}_{4}\right)$. The solvent was evaporated under vacuo and the residue was purified by flash column chromatography (ethyl acetate/cyclohexane, 3:2) giving $(S)-13$ as a colourless oil $(1.179 \mathrm{~g}, 79 \%) . R_{f} 0.60$ (EtOAc/cyclohexane, $\left.4 / 1\right) ;[\alpha]_{\mathrm{D}}{ }^{25}=+4.0$ (c $\left.0.75, \mathrm{CHCl}_{3}\right)\left(\right.$ lit. $^{17}[\alpha]_{\mathrm{D}}{ }^{25}=+6.3\left(\mathrm{c} 0.5, \mathrm{CHCl}_{3}\right)$ ); IR (neat) $v 3360,1733,1691$; ${ }^{1} \mathrm{H} \mathrm{NMR}$ $\left(\mathrm{CDCl}_{3}\right) \delta 5.21(\mathrm{bs}, 1 \mathrm{H}, \mathrm{NH}), 3.98(\mathrm{~m}, 1 \mathrm{H}, \mathrm{CH}), 3.71\left(\mathrm{t}, J=5.5 \mathrm{~Hz}, 2 \mathrm{H}, \mathrm{CH}_{2}-\mathrm{OH}\right), 3.70(\mathrm{~s}, 3 \mathrm{H}$, $\left.\mathrm{O}-\mathrm{CH}_{3}\right), 2.64\left(\mathrm{~d}, J=6.0 \mathrm{~Hz}, 2 \mathrm{H}, \mathrm{CH}_{2}\right), 2.48(\mathrm{bs}, 1 \mathrm{H}, \mathrm{OH}), 1.44(\mathrm{~s}, 9 \mathrm{H}, t-\mathrm{Bu}) ;{ }^{13} \mathrm{C} \mathrm{NMR}\left(\mathrm{CDCl}_{3}\right)$ $\delta 172.2\left(\mathrm{CO}_{2} \mathrm{Me}\right), 155.7\left(\mathrm{CO}_{2} t-\mathrm{Bu}\right), 79.6(\mathrm{C}), 63.8\left(\mathrm{CH}_{2}-\mathrm{O}\right), 51.6\left(\mathrm{O}_{-} \mathrm{CH}_{3}\right), 49.2(\mathrm{CH}), 35.7$ $\left(\mathrm{CH}_{2}\right), 28.2(t$-Bu $)$; HRMS-ESI $m / z$ calcd for $\mathrm{C}_{10} \mathrm{H}_{19} \mathrm{NO}_{5} \mathrm{Na}[\mathrm{M}+\mathrm{Na}]^{+}:$256.1161, found:256.1169.

Methyl (3S)-3-[(tert-butoxycarbonyl)amino]-4-(tert-butyldiphenylsilyloxy)butanoate (14a). To a stirred solution of $(S)-13(1.43 \mathrm{~g}, 6.1 \mathrm{mmol})$ in anhydrous dichloromethane $(50 \mathrm{~mL})$ at $0{ }^{\circ} \mathrm{C}$ and under argon atmosphere was added imidazole $(0.63 \mathrm{~g}, 9.2 \mathrm{mmol})$ followed $15 \mathrm{~min}$ later by tert-butyldiphenylsilyl chloride $(90 \mathrm{~mL}, 7.3 \mathrm{mmol})$. The reaction mixture was slowly allowed to reach $20{ }^{\circ} \mathrm{C}(3 \mathrm{~h})$ and stirring was continued for $1 \mathrm{~h}$ before dilution by diethyl ether. The organic layer was washed with water $(30 \mathrm{~mL})$, brine $(20 \mathrm{~mL})$ and dried $\left(\mathrm{MgSO}_{4}\right)$. The solvent was removed under reduced pressure and the residue was purified by column chromatography (diethyl ether/pentane, 1:4) to give 14a as a colourless oil (2.82 $\mathrm{g}, 98 \%$ ). $R_{f} 0.60$ (EtOAc/cyclohexane 3/7); $[\alpha]_{\mathrm{D}}{ }^{25}=-7.9$ (c 2.1, $\mathrm{CHCl}_{3}$ ); IR (neat) $v_{\max } 3434,1745,1720 ;{ }^{1} \mathrm{H}$ NMR $\left(\mathrm{CDCl}_{3}\right) \delta 7.64(\mathrm{~d}, J=8.0 \mathrm{~Hz}, 4 \mathrm{H}, \mathrm{H}-\mathrm{Ar}), 7.45-7.37$ (m, 6H, H-Ar), 5.09 (d, $J=7.5 \mathrm{~Hz}$, $1 \mathrm{H}, \mathrm{NH}), 4.12(\mathrm{~m}, 1 \mathrm{H}, \mathrm{CH}), 3.72\left(\mathrm{~m}, 2 \mathrm{H}, \mathrm{CH}_{2}-\mathrm{O}\right), 3.64\left(\mathrm{~s}, 3 \mathrm{H}, \mathrm{O}-\mathrm{CH}_{3}\right), 2.66\left(\mathrm{~m}, 2 \mathrm{H}, \mathrm{CH}_{2}\right), 1.44$ $(\mathrm{s}, 9 \mathrm{H}, t-\mathrm{Bu}), 1.07(\mathrm{~s}, 9 \mathrm{H}, t-\mathrm{Bu}) ;{ }^{13} \mathrm{C} \mathrm{NMR}\left(\mathrm{CDCl}_{3}\right) \delta 171.8\left(\mathrm{CO}_{2} \mathrm{Me}\right), 155.1\left(\mathrm{CO}_{2} t-\mathrm{Bu}\right), 135.5$ (C-Ar), 133.1 (C-Ar), 133.0 (C-Ar), 129.8 (C-Ar), 127.7 (C-Ar), 79.3 (C), 64.9 ( $\left.\mathrm{CH}_{2}-\mathrm{O}\right), 51.6$ $\left(\mathrm{O}-\mathrm{CH}_{3}\right), 48.8(\mathrm{CH}), 35.9\left(\mathrm{CH}_{2}\right), 28.3(t-\mathrm{Bu}), 26.8(t-\mathrm{Bu}), 19.2(\mathrm{C})$; $\mathrm{MS}(\mathrm{CI}+) \mathrm{m} / z 472([\mathrm{M}+$ $\left.\mathrm{H}]^{+}, 20\right), 427$ (20), 372 (100), 294 (11); HRMS-ESI $m / z$ calcd for $\mathrm{C}_{26} \mathrm{H}_{37} \mathrm{NO}_{5} \mathrm{SiNa}[\mathrm{M}+\mathrm{Na}]^{+}$: 494.2339, found:494.2342.

Methyl (3S)-3-[(bis(tert-butoxycarbonyl)amino]-4-(tert-butyldiphenylsilyl)oxy)-butanoate (14b). To a stirred solution of $(S)-14 a(0.598 \mathrm{~g}, 1.31 \mathrm{mmol})$ in anhydrous acetonitrile $(5 \mathrm{~mL})$ were successively added, at $20^{\circ} \mathrm{C}$, 4-dimethylaminopyridine $(0.032 \mathrm{~g}, 0.26 \mathrm{mmol})$ and di-tertbutyl dicarbonate $(0.314 \mathrm{~g}, 1.44 \mathrm{mmol})$. After $12 \mathrm{~h}$, additionnal di-tert-butyl dicarbonate $(0.314$ $\mathrm{g}, 1.44 \mathrm{mmol}$ ) was added to the mixture and the reaction was continued for another $12 \mathrm{~h}$. The solvent was then evaporated under reduced pressure and the crude residue was purified by column chromatography (ethyl acetate/cyclohexane, 1:9) to give $\mathbf{1 4 b}$ as an oil (409 mg, 54\%). $R_{f}$ $0.44($ EtOAc/cyclohexane $1 / 9) ;[\alpha]_{\mathrm{D}}^{25}=-7.18\left(\mathrm{c}=1.38, \mathrm{CHCl}_{3}\right)$; IR (neat) $v_{\max } 2980,2932$, 
2859, 1744, 1703; ${ }^{1} \mathrm{H}$ NMR $\left(\mathrm{CDCl}_{3}\right) \delta$ 7.69-7.65 (m, 4H, H-Ar), 7.43-7.35 (m, 6H, H-Ar), 4.91 (quint, $J=8.0 \mathrm{~Hz}, 1 \mathrm{H}, \mathrm{CH}), 3.94\left(\mathrm{dd}, J=9.5,8.0 \mathrm{~Hz}, 1 \mathrm{H}, \mathrm{CH}_{2}-\mathrm{O}\right), 3.73(\mathrm{dd}, J=9.5,7.0 \mathrm{~Hz}, 1 \mathrm{H}$, $\mathrm{CH}_{2}-\mathrm{O}$ ), 3.64 (s, 3H, O-CH$)_{3}, 2.84$ (dd, $J=15.5,8.5 \mathrm{~Hz}, 1 \mathrm{H}, \mathrm{CH}_{2}$ ), 2.64 (dd, $J=16.0,6.0 \mathrm{~Hz}$, $\left.1 \mathrm{H}, \mathrm{CH}_{2}\right), 1.48(\mathrm{~s}, 18 \mathrm{H}, t-\mathrm{Bu}), 1.04(\mathrm{~s}, 9 \mathrm{H}, t-\mathrm{Bu}) ;{ }^{13} \mathrm{C} \mathrm{NMR}\left(\mathrm{CDCl}_{3}\right) \delta 171.5\left(\mathrm{CO}_{2} \mathrm{Me}\right), 152.9$ $\left(\mathrm{CO}_{2} t \mathrm{Bu}\right), 135.5$ (C-Ar), 133.3 (C-Ar), 129.6 (C-Ar), 127.6 (C-Ar), $82.2(\mathrm{C}), 63.8\left(\mathrm{CH}_{2}-\mathrm{O}\right), 55.1$ $\left(\mathrm{O}-\mathrm{CH}_{3}\right), 51.6(\mathrm{CH}), 35.1\left(\mathrm{CH}_{2}\right), 28.0(t-\mathrm{Bu}), 26.6(t$-Bu $), 19.1(\mathrm{C})$; HRMS-ESI $m / z$ calcd for $\mathrm{C}_{31} \mathrm{H}_{45} \mathrm{NO}_{7} \mathrm{SiNa}[\mathrm{M}+\mathrm{Na}]^{+}: 594.2863$, found: 594.2844 .

Methyl (3S)-3-[benzyl(tert-butoxycarbonyl)amino]-4-(tert-butyldiphenylsilyloxy)butanoate (14c). A stirred solution of $(S)-14 a(1.00 \mathrm{~g}, 2.10 \mathrm{mmol})$ in anhydrous dimethylformamide (10 $\mathrm{mL}$ ) at $0{ }^{\circ} \mathrm{C}$ and under argon atmosphere, was treated with benzyl bromide (377 $\left.\mu 1,3.15 \mathrm{mmol}\right)$ and tetrabutylammonium iodide $(1.16 \mathrm{~g}, 3.15 \mathrm{mmol})$ followed by a stirred suspension of sodium hydride $(60 \%$ dispersion in mineral oil, $0.168 \mathrm{~g}, 4.20 \mathrm{mmol})$ in anhydrous dimethylformamide $(2$ $\mathrm{mL}$ ) which was dropwise transferred via a cannula. The reaction was allowed to stir at $0{ }^{\circ} \mathrm{C}$ for 1 $\mathrm{h}$ before being stopped by adding saturated aqueous ammonium chloride solution $(10 \mathrm{~mL})$ and extracted twice with diethyl ether $(20 \mathrm{~mL})$. The organic layer was washed with brine, dried $\left(\mathrm{MgSO}_{4}\right)$, concentrated under reduced pressure and purified by column chromatography (ethyl acetate/cyclohexane, 1:9) to give 14c as a pale yellow oil (0.815 g, 69\%). Compound 14c appeared in the NMR spectra at room temperature as a mixture of two rotamers $a$ and $b . R_{f} 0.51$ (EtOAc/cyclohexane 1/4); $[\alpha]_{\mathrm{D}}{ }^{25}=-20.5\left(\mathrm{c}=2.42, \mathrm{CHCl}_{3}\right)$; IR (neat) $v_{\max } 1740,1695 ;{ }^{1} \mathrm{H} \mathrm{NMR}$ $\left(\mathrm{CDCl}_{3}\right) \delta 7.81-7.28(\mathrm{~m}, 15 \mathrm{H}, \mathrm{H}-\mathrm{Ar}), 4.72\left(\mathrm{~m}, 1 \mathrm{H}, \mathrm{CH}_{2} \mathrm{Ph}\right), 4.49\left(\mathrm{~m}, 2 \mathrm{H}, \mathrm{CH}_{2} \mathrm{Ph}, \mathrm{CH}, a\right), 4.29$ $(\mathrm{m}, 1 \mathrm{H}, \mathrm{CH}, b), 3.86\left(\mathrm{~m}, 1 \mathrm{H}, \mathrm{CH}_{2}-\mathrm{O}\right), 3.74\left(\mathrm{~m}, 1 \mathrm{H}, \mathrm{CH}_{2}-\mathrm{O}\right), 3.60$ (s, 3H, O-CH $), 2.76(\mathrm{~m}, 2 \mathrm{H}$, $\left.\mathrm{CH}_{2}\right), 1.50(\mathrm{~s}, 9 \mathrm{H}, t \mathrm{Bu}, a), 1.46(\mathrm{~s}, 9 \mathrm{H}, t-\mathrm{Bu}, b), 1.14(\mathrm{~s}, 9 \mathrm{H}, t-\mathrm{Bu}, b), 1.10(\mathrm{~s}, 9 \mathrm{H}, t \mathrm{Bu}, a) ;{ }^{13} \mathrm{C}$ $\operatorname{NMR}\left(\mathrm{CDCl}_{3}\right) \delta 171.9\left(\mathrm{CO}_{2} \mathrm{Me}, a\right.$ and $\left.b\right), 155.6\left(\mathrm{CO}_{2} t-\mathrm{Bu}, a\right), 155.1\left(\mathrm{CO}_{2} t-\mathrm{Bu}, b\right), 138.8(\mathrm{C}-\mathrm{Ar}$, a), 135.4 (C-Ar, $a$ and $b$ ), 133.0 (C-Ar, $a$ and $b$ ), 132.9 (C-Ar, $a$ and $b$ ), 129.7 (C-Ar, $a$ ), 129.6 (C-Ar, $a$ and $b$ ), 129.3 (C-Ar, $b$ ), 128.3 (C-Ar, $a$ ), 127.6 (C-Ar, $a$ and $b$ ), 80.2 (C, $a), 79.8$ (C, $b$ ), $64.6\left(\mathrm{CH}_{2}-\mathrm{O}, a\right), 64.2\left(\mathrm{CH}_{2}-\mathrm{O}, b\right), 56.1(\mathrm{CH}, a$ and $b), 51.5\left(\mathrm{O}-\mathrm{CH}_{3}\right), 50.5\left(\mathrm{CH}_{2}-\mathrm{Ph}, a\right.$ and $\left.b\right)$, $35.6\left(\mathrm{CH}_{2}, a\right), 34.8\left(\mathrm{CH}_{2}, b\right), 28.2(t-\mathrm{Bu}, a$ and $b), 26.7(t-\mathrm{Bu}, a$ and $b), 19.0(\mathrm{C})$; MS (ESI) $m / z$ $584\left([\mathrm{M}+\mathrm{Na}]^{+}, 62\right), 462(100), 310(30), 141$ (27); HRMS-ESI $m / z$ calcd for $\mathrm{C}_{33} \mathrm{H}_{43} \mathrm{NO}_{5} \mathrm{SiNa}$ $[\mathrm{M}+\mathrm{Na}]^{+}: 584.2808$, found: 584.2794 .

Methyl [(4S)-3-(tert-butoxycarbonyl)-2,2-dimethyl-1,3-oxazolidin-4-yl]ethanoate (14d). Compound 14d was prepared from $(S)$-13 following the general procedure for preparation of oxazolidines and appeared in the NMR spectra at ambient temperature as a mixture of two rotamers $a$ and $b$. Colourless oil $(0.826 \mathrm{~g}, 87 \%)$. $R_{f} 0.64$ (EtOAc/cyclohexane, $\left.1: 1\right) ;[\alpha]_{\mathrm{D}}{ }^{25}=+$ $28.2\left(\mathrm{c} 0.54, \mathrm{CHCl}_{3}\right)\left(\right.$ lit. $\left.^{15}[\alpha]_{\mathrm{D}}{ }^{25}=+27.9\left(\mathrm{c} 1.0, \mathrm{CHCl}_{3}\right)\right)$; IR (neat): $v_{\max } 1740,1700 ;{ }^{1} \mathrm{H} \mathrm{NMR}$ $\left(\mathrm{CDCl}_{3}\right) \delta 4.26(\mathrm{~m}, 1 \mathrm{H}, \mathrm{CH}, a), 4.15(\mathrm{~m}, 1 \mathrm{H}, \mathrm{CH}, b), 4.00\left(\mathrm{~m}, 2 \mathrm{H}, \mathrm{CH}_{2}-\mathrm{O}, a\right.$ and $\left.b\right), 3.82(\mathrm{~d}, J=$ $\left.9.0 \mathrm{~Hz}, 1 \mathrm{H}, \mathrm{CH}_{2}-\mathrm{O}, a\right), 3.82\left(\mathrm{~d}, J=9.0 \mathrm{~Hz}, 1 \mathrm{H}, \mathrm{CH}_{2}-\mathrm{O}, b\right), 3.67$ (s, 3H, O-CH $\left.3, b\right), 3.64$ (s, 3H, O-CH $3, a), 2.91$ (bd, $\left.J=15.5 \mathrm{~Hz}, 1 \mathrm{H}, \mathrm{CH}_{2}, a\right), 2.74$ (bd, $\left.J=15.5 \mathrm{~Hz}, 1 \mathrm{H}, \mathrm{CH}_{2}, b\right), 2.52$ (dd, $J=$ 15.5, $\left.11.0 \mathrm{~Hz}, 1 \mathrm{H}, \mathrm{CH}_{2}, b\right), 2.47\left(\mathrm{dd}, J=15.5,11.0 \mathrm{~Hz}, 1 \mathrm{H}, \mathrm{CH}_{2}, a\right), 1.56\left(\mathrm{~s}, 3 \mathrm{H}, \mathrm{CH}_{3}, b\right), 1.52$ $\left(\mathrm{s}, 3 \mathrm{H}, \mathrm{CH}_{3}, a\right), 1.46\left(\mathrm{~s}, 18 \mathrm{H}, \mathrm{CH}_{3}\right.$ and $t-\mathrm{Bu} a$ and $\left.b\right), 1.45\left(\mathrm{~s}, 6 \mathrm{H}, \mathrm{CH}_{3}, a\right.$ and $\left.b\right) ;{ }^{13} \mathrm{C}$ NMR $\left(\mathrm{CDCl}_{3}\right) \delta 171.7\left(\mathrm{CO}_{2} \mathrm{Me}, a\right.$ and $\left.b\right), 151.9\left(\mathrm{CO}_{2} t-\mathrm{Bu}, a\right), 151.3\left(\mathrm{CO}_{2} t-\mathrm{Bu}, b\right), 93.8(\mathrm{C}, b), 93.3(\mathrm{C}$, 
a), $80.4(\mathrm{C}, a), 80.0(\mathrm{C}, b), 67.5\left(\mathrm{CH}_{2}-\mathrm{O}, b\right), 67.3\left(\mathrm{CH}_{2}-\mathrm{O}, a\right), 54.2(\mathrm{CH}, a), 53.9(\mathrm{CH}, b), 51.6$ $\left(\mathrm{O}-\mathrm{CH}_{3}, a\right.$ and $\left.b\right), 37.7\left(\mathrm{CH}_{2}, b\right), 36.9\left(\mathrm{CH}_{2}, a\right), 28.4(t-\mathrm{Bu}, a$ and $b), 27.6\left(\mathrm{CH}_{3}, a\right), 26.7\left(\mathrm{CH}_{3}\right.$, b), $24.4\left(\mathrm{CH}_{3}, a\right), 23.1\left(\mathrm{CH}_{3}, b\right)$.

tert-Butyl $N$-[(1S)-1-[(tert-butyldiphenylsilyloxy)methyl]-3-hydroxypropyl]carbamate (15a). Compound 15a was prepared from $(S)$-14a following the general method A of reduction. Colourless oil (0.452 g, 98\%). $R_{f} 0.58$ (EtOAc/cyclohexane, 1:1); $[\alpha]_{\mathrm{D}}{ }^{25}=-6.7$ (c 2.0, $\mathrm{CHCl}_{3}$ ); IR (neat) $v_{\max } 3440,1695 ;{ }^{1} \mathrm{H}$ NMR $\left(\mathrm{CDCl}_{3}\right) \delta 7.63(\mathrm{~d}, J=7.0 \mathrm{~Hz}, 4 \mathrm{H}, \mathrm{H}-\mathrm{Ar}), 7.45$ (t, $J=7.0$ $\mathrm{Hz}, 2 \mathrm{H}, \mathrm{H}-\mathrm{Ar}$ ), 7.40 (t, $J=7.0 \mathrm{~Hz}, 4 \mathrm{H}, \mathrm{H}-\mathrm{Ar}), 4.83$ (d, $J=9.0 \mathrm{~Hz}, 1 \mathrm{H}, \mathrm{NH}), 3.87$ (m, 1H, CH), $3.80\left(\mathrm{dd}, J=10.5,3.5 \mathrm{~Hz}, 1 \mathrm{H}, \mathrm{CH}_{2}-\mathrm{O}\right), 3.66\left(\mathrm{~m}, 2 \mathrm{H}, \mathrm{CH}_{2}-\mathrm{OH}\right), 3.62(\mathrm{dd}, J=10.5,3.5 \mathrm{~Hz}, 1 \mathrm{H}$, $\left.\mathrm{CH}_{2}-\mathrm{O}\right), 3.42(\mathrm{bs}, 1 \mathrm{H}, \mathrm{OH}), 1.72\left(\mathrm{~m}, 1 \mathrm{H}, \mathrm{CH}_{2}\right), 1.63\left(\mathrm{~m}, 1 \mathrm{H}, \mathrm{CH}_{2}\right), 1.46(\mathrm{~s}, 9 \mathrm{H}, t-\mathrm{Bu}), 1.08(\mathrm{~s}$, $9 \mathrm{H}, t \mathrm{Bu}) ;{ }^{13} \mathrm{C}$ NMR $\left(\mathrm{CDCl}_{3}\right) \delta 157.0\left(\mathrm{CO}_{2} t \mathrm{-Bu}\right), 135.5$ (C-Ar), $133.1(\mathrm{C}-\mathrm{Ar}), 133.0(\mathrm{C}-\mathrm{Ar})$, 129.8 (C-Ar), 127.8 (C-Ar), $79.7(\mathrm{C}), 66.3\left(\mathrm{CH}_{2}-\mathrm{O}\right), 58.7\left(\mathrm{CH}_{2}-\mathrm{OH}\right), 48.6(\mathrm{CH}), 35.3\left(\mathrm{CH}_{2}\right)$, $28.3(t-\mathrm{Bu}), 26.8(t-\mathrm{Bu}), 19.3(\mathrm{C})$; HRMS-ESI $\mathrm{m} / z$ calcd for $\mathrm{C}_{25} \mathrm{H}_{37} \mathrm{NO}_{4} \mathrm{SiNa}[\mathrm{M}+\mathrm{Na}]^{+}$: 466.2390, found: 466.2369 .

tert-Butyl $\quad N$-[(1S)-1-[(tert-butyldiphenylsilyloxy)methyl]-3-hydroxypropyl]bis-carbamate (15b). To a stirred solution of $(S)$-14b $(0.401 \mathrm{~g}, 0.70 \mathrm{mmol})$ in anhydrous ether $(5 \mathrm{~mL})$, at - 78 ${ }^{\circ} \mathrm{C}$ and under argon atmosphere, was added a $1.0 \mathrm{M}$ solution of diisobutylaluminium hydride in hexane $(770 \mu \mathrm{L}, 0.77 \mathrm{mmol})$. After $45 \mathrm{~min}$ the reaction was quenched by adjunction of saturated aqueous potassium and aqueous sodium tartaric acid salts solution $(5 \mathrm{~mL})$. The aqueous portion was extracted with diethyl ether. The combined organic layers were washed by brine before drying $\left(\mathrm{MgSO}_{4}\right)$. The solvent was then evaporated under reduced pressure and the crude residue was purified by column chromatography (ethyl acetate/cyclohexane, $2: 8$ ) to give $0.375 \mathrm{~g}$ of ditert-butyl [1-[(tert-butyldiphenylsilyloxy)methyl]-3-oxopropyl]imidodicarbonate which was dissolved in absolute methanol $(4 \mathrm{~mL})$ at $0{ }^{\circ} \mathrm{C}$. Then, sodium borohydride $(0.053 \mathrm{~g}, 1.40 \mathrm{mmol})$ was added. Stirring was continued for an additional $30 \mathrm{~min}$. The reaction was quenched by saturated aqueous ammonium chloride solution $(4 \mathrm{~mL})$ and the reaction mixture was diluted with ether $(5 \mathrm{~mL})$. The organic layer was separated, washed with brine and dried $\left(\mathrm{MgSO}_{4}\right)$. The solvent was concentrated under reduced pressure and the residue was purified by column chromatography (ethyl acetate/cyclohexane, 1:4) to give $\mathbf{1 5 b}$ as a colourless oil $(0.320 \mathrm{~g}, 84 \%)$. $R_{f} 0.44$ (EtOAc/cyclohexane, 1:4); $[\alpha]_{\mathrm{D}}^{25}=+15.34\left(\mathrm{c} 0.91, \mathrm{CHCl}_{3}\right.$ ); IR (neat): $v_{\max } 3501,1738$, 1699; ${ }^{1} \mathrm{H}$ NMR $\left(\mathrm{CDCl}_{3}\right) \delta$ 7.68-7.64 (m, 4H, H-Ar), 7.44-7.35 (m, 6H, H-Ar), 4.43 (m, 1H, CH), 4.13 (dd, $J=10.0,8.5 \mathrm{~Hz}, 1 \mathrm{H}, \mathrm{CH}_{2}-\mathrm{O}$ ), 3.66-3.58 (m, 3H, $\mathrm{CH}_{2}, \mathrm{CH}_{2}-\mathrm{O}$ ), 2.94 (bs, $\left.1 \mathrm{H}, \mathrm{OH}\right), 1.85$ $\left(\mathrm{m}, 1 \mathrm{H}, \mathrm{CH}_{2}\right), 1.68\left(\mathrm{~m}, 1 \mathrm{H}, \mathrm{CH}_{2}\right), 1.49(\mathrm{~s}, 18 \mathrm{H}, t-\mathrm{Bu}), 1.04(\mathrm{~s}, 9 \mathrm{H}, t-\mathrm{Bu}) ;{ }^{13} \mathrm{C} \mathrm{NMR}\left(\mathrm{CDCl}_{3}\right) \delta$ $154.1\left(\mathrm{CO}_{2} t \mathrm{Bu}\right), 135.5$ (C-Ar) 133.4 (C-Ar), $129.6(\mathrm{C}-\mathrm{Ar}), 127.6(\mathrm{C}-\mathrm{Ar}), 82.5(\mathrm{C}), 64.8\left(\mathrm{CH}_{2}-\mathrm{O}\right)$, $59.0\left(\mathrm{CH}_{2}-\mathrm{OH}\right), 56.8(\mathrm{CH}), 31.6\left(\mathrm{CH}_{2}\right), 28.0(t$-Bu $), 26.7(t$-Bu $), 19.2(\mathrm{C})$; HRMS-ESI $m / z$ calcd for $\mathrm{C}_{30} \mathrm{H}_{45} \mathrm{NO}_{6} \mathrm{SiNa}[\mathrm{M}+\mathrm{Na}]^{+}: 566.2914$, found: 566.2894 .

tert-Butyl $N$-benzyl- $N$-[(1S)-1-[(tert-butyldiphenylsilyloxy)methyl]-3-hydroxypropyl]carbamate (15c). Compound 15c was prepared from $(S)-\mathbf{1 4 c}$ using the general method A of reduction. Colourless oil (0.550 mg, 99\%). $R_{f} 0.49$ (EtOAc/cyclohexane, 3/7); $[\alpha]_{\mathrm{D}}^{25}=+2.4$ (c 1.0, $\mathrm{CHCl}_{3}$ ); IR (neat) $v_{\max } 3437,1684 ;{ }^{1} \mathrm{H}$ NMR $\left(\mathrm{CDCl}_{3}\right) \delta 7.60-7.21(\mathrm{~m}, 15 \mathrm{H}, \mathrm{H}-\mathrm{Ar}), 4.48\left(\mathrm{~d}, J=16.0 \mathrm{~Hz}, 1 \mathrm{H}, \mathrm{CH}_{2^{-}}\right.$ 
$\mathrm{Ph}), 4.43(\mathrm{~m}, 1 \mathrm{H}, \mathrm{CH}), 4.34\left(\mathrm{~d}, J=16.0 \mathrm{~Hz}, 1 \mathrm{H}, \mathrm{CH}_{2}-\mathrm{Ph}\right), 3.74\left(\mathrm{~m}, 2 \mathrm{H}, \mathrm{CH}_{2}-\mathrm{O}\right), 3.45$ (m, 2H, $\left.\mathrm{CH}_{2}-\mathrm{OH}\right), 3.22$ (bs, 1H, OH), 1.60-1.35 (m, 2H, $\left.\mathrm{CH}_{2}\right), 1.42$ (s, 9H, $t$-Bu), 1.39 (s, 9H, $t$-Bu), 1.03 $\left(\mathrm{s}, 9 \mathrm{H}, t\right.$-Bu); ${ }^{13} \mathrm{C} \mathrm{NMR}\left(\mathrm{CDCl}_{3}\right) \delta 157.3\left(\mathrm{CO}_{2} t\right.$-Bu), 139.5 (C-Ar), 135.5 (C-Ar), 133.1 (C-Ar), 133.0 (C-Ar), 129.7 (C-Ar), 128.2 (C-Ar), 127.7 (C-Ar), 126.9 (C-Ar), 126.7 (C-Ar), 80.3 (C), $64.7\left(\mathrm{CH}_{2}-\mathrm{O}\right), 58.6\left(\mathrm{CH}_{2}-\mathrm{OH}\right), 54.1(\mathrm{CH}), 47.3\left(\mathrm{CH}_{2}-\mathrm{Ph}\right), 32.0\left(\mathrm{CH}_{2}\right), 28.2(t-\mathrm{Bu}), 26.8(t-\mathrm{Bu})$, 19.1 (C); MS (ESI) $m / z 556\left([\mathrm{M}+\mathrm{Na}]^{+}, 100\right), 434$ (100); HRMS-ESI $\mathrm{m} / z$ calcd for $\mathrm{C}_{32} \mathrm{H}_{43} \mathrm{NO}_{4} \mathrm{SiNa}[\mathrm{M}+\mathrm{Na}]^{+}:$556.2859, found: 556.2861.

tert-Butyl [(4S)-4-(2-hydroxyethyl)-2,2-dimethyl-1,3-oxazolidin-3-yl]carboxylate (15d). Compound 15d was prepared from $(S)$-14d using the general method $\mathrm{C}$ of reduction. White needles (0.473 g, quant.); $m p 75{ }^{\circ} \mathrm{C}$ (pentane); $R_{f} 0.46$ (EtOAc/cyclohexane, $\left.7 / 3\right) ;[\alpha]_{\mathrm{D}}{ }^{25}=-12.2$ (c $\left.1.06, \mathrm{CHCl}_{3}\right)\left(\right.$ lit. $^{18 \mathrm{~b}}[\alpha]_{\mathrm{D}}{ }^{25}=-12.3\left(\mathrm{c} 1.53, \mathrm{CHCl}_{3}\right)$ ); IR $(\mathrm{KBr}) v_{\max } 3260,1690$; ${ }^{1} \mathrm{H}$ NMR $\left(\mathrm{CDCl}_{3}\right) \delta 4.20(\mathrm{~m}, 1 \mathrm{H}, \mathrm{CH}), 3.99\left(\mathrm{~m}, 2 \mathrm{H}, \mathrm{H}-4, \mathrm{CH}_{2}-\mathrm{OH}\right), 3.68\left(\mathrm{~d}, J=9.0 \mathrm{~Hz}, 1 \mathrm{H}, \mathrm{CH}_{2}-\mathrm{O}\right), 3.63$ (bs, $1 \mathrm{H}, \mathrm{OH}$ ), 3.53 (bt, $\left.J=10.5 \mathrm{~Hz}, 1 \mathrm{H}, \mathrm{CH}_{2}-\mathrm{OH}\right), 1.81$ (m, $1 \mathrm{H}, \mathrm{CH}_{2}$ ), 1.71 (ddt, $J=13.5,10.5$, $\left.3.0 \mathrm{~Hz}, 1 \mathrm{H}, \mathrm{CH}_{2}\right), 1.53\left(\mathrm{~s}, 3 \mathrm{H}, \mathrm{CH}_{3}\right), 1.48\left(\mathrm{~s}, 12 \mathrm{H}, \mathrm{CH}_{3}\right.$ and $\left.t-\mathrm{Bu}\right) ;{ }^{13} \mathrm{C} \mathrm{NMR}\left(\mathrm{CDCl}_{3}\right) \delta 153.8$ $\left(\mathrm{CO}_{2} t-\mathrm{Bu}\right), 93.6(\mathrm{C}), 80.9(\mathrm{C}), 68.2\left(\mathrm{CH}_{2}-\mathrm{O}\right), 58.6\left(\mathrm{CH}_{2}-\mathrm{OH}\right), 54.0(\mathrm{CH}), 37.7\left(\mathrm{CH}_{2}\right), 28.3(t-$ $\mathrm{Bu}), 27.7\left(\mathrm{CH}_{3}\right), 24.3\left(\mathrm{CH}_{3}\right)$.

tert-Butyl $\quad \mathrm{N}$-[(1S)-1-[(tert-butyldiphenylsilyloxy)methyl]-3-iodopropyl]carbamate (16a). Compound 16a was obtained from $(S)$-15a following the general procedure for preparation of iodides using toluene as solvent. Pale yellow foam $(520 \mathrm{mg}, 92 \%) ; R_{f} 0.44$ (EtOAc/cyclohexane $1 / 9$ ); $m p$ 57-58 ${ }^{\circ} \mathrm{C}$ (pentane); $[\alpha]_{\mathrm{D}}{ }^{25}=-16.9$ (c 2.46, $\mathrm{CHCl}_{3}$ ); IR (KBr) $v_{\max } 3440,1707 ;{ }^{1} \mathrm{H}$ NMR $\left(\mathrm{CDCl}_{3}\right) \delta 7.63$ (m, 4H, H-Ar), 7.42 (m, 6H, H-Ar), 4.71 (d, J=8.5 Hz, 1H, NH), 3.73 (m, $1 \mathrm{H}, \mathrm{CH}), 3.69$ (dd, $\left.J=10.0,2.5 \mathrm{~Hz}, 1 \mathrm{H}, \mathrm{CH}_{2}-\mathrm{O}\right), 3.60$ (bd, $\left.J=10.0 \mathrm{~Hz}, 1 \mathrm{H}, \mathrm{CH}_{2}-\mathrm{O}\right), 3.13$ (m, $\left.2 \mathrm{H}, \mathrm{CH}_{2}-\mathrm{I}\right), 2.10\left(\mathrm{q}, J=6.5 \mathrm{~Hz}, 2 \mathrm{H}, \mathrm{CH}_{2}\right), 1.45(\mathrm{~s}, 9 \mathrm{H}, t-\mathrm{Bu}), 1.07(\mathrm{~s}, 9 \mathrm{H}, t-\mathrm{Bu}) ;{ }^{13} \mathrm{C} \mathrm{NMR}$ $\left(\mathrm{CDCl}_{3}\right) \delta 155.5\left(\mathrm{CO}_{2} t-\mathrm{Bu}\right), 135.5(\mathrm{C}-\mathrm{Ar}), 133.0$ (C-Ar), $133.0(\mathrm{C}-\mathrm{Ar}), 129.9(\mathrm{C}-\mathrm{Ar}), 127.8(\mathrm{C}-$ Ar), $79.4(\mathrm{C}), 65.2\left(\mathrm{CH}_{2}-\mathrm{O}\right), 52.8(\mathrm{CH}), 36.7\left(\mathrm{CH}_{2}\right), 28.4(t-\mathrm{Bu}), 26.9(t-\mathrm{Bu}), 19.3(\mathrm{C}), 1.5\left(\mathrm{CH}_{2^{-}}\right.$ I); MS (ESI) $m / z 592\left([\mathrm{M}+\mathrm{K}]^{+}, 100\right), 576\left([\mathrm{M}+\mathrm{Na}]^{+}, 92\right), 454(\mathrm{M}-\mathrm{Boc}, 26) ;$ HRMS-ESI $m / z$ calcd for $\mathrm{C}_{25} \mathrm{H}_{36} \mathrm{INO}_{3} \mathrm{SiNa}[\mathrm{M}+\mathrm{Na}]^{+}:$576.1407, found: 576.1421 .

Di-tert-butyl $N$-[(1S)-1-[(tert-butyldiphenylsilyloxy)methyl]-3-iodopropyl]imidodicarbonate (16b). Compound 16b was obtained from $(S)$-15b following the general procedure for preparation of iodides in diethyl ether/acetonitrile. Colourless oil (613 mg, 92\%). $R_{f} 0.56$ (EtOAc/cyclohexane, 1/19); $[\alpha]_{\mathrm{D}}{ }^{25}=-12.08\left(\mathrm{c} 0.76, \mathrm{CHCl}_{3}\right)$; IR (neat) $v_{\max } 3069,2979,2931$, 2859, 1739, 1701, 1428; ${ }^{1} \mathrm{H}$ NMR $\left(\mathrm{CDCl}_{3}\right) \delta 7.63$ (m, 4H, H-Ar), 7.42 (m, 6H, H-Ar), 4.48 (m, $1 \mathrm{H}, \mathrm{CH}), 3.94\left(\mathrm{dd}, J=10.0,8.5 \mathrm{~Hz}, 1 \mathrm{H}, \mathrm{CH}_{2}-\mathrm{O}\right), 3.66\left(\mathrm{dd}, J=10.0,6.5 \mathrm{~Hz}, 1 \mathrm{H}, \mathrm{CH}_{2}-\mathrm{O}\right), 3.21-$ $3.08\left(\mathrm{~m}, 2 \mathrm{H}, \mathrm{CH}_{2} \mathrm{I}\right), 2.33\left(\mathrm{~m}, 1 \mathrm{H}, \mathrm{CH}_{2}\right), 2.08\left(\mathrm{~m}, 1 \mathrm{H}, \mathrm{CH}_{2}\right), 1.48(\mathrm{~s}, 9 \mathrm{H}, t \mathrm{Bu}), 1.04(\mathrm{~s}, 9 \mathrm{H}, t-\mathrm{Bu})$; ${ }^{13} \mathrm{C} \mathrm{NMR}\left(\mathrm{CDCl}_{3}\right) \delta 153.1\left(\mathrm{CO}_{2} t\right.$-Bu), 135.5 (C-Ar), 133.4 (C-Ar), 129.7 (C-Ar), $129.6(\mathrm{C}-\mathrm{Ar})$, 127.7 (C-Ar), 127.6 (C-Ar), $82.3(\mathrm{C}), 64.1\left(\mathrm{CH}_{2}-\mathrm{O}\right), 59.4(\mathrm{CH}), 34.2\left(\mathrm{CH}_{2}\right), 28.0(t-\mathrm{Bu}), 26.7(t-$ $\mathrm{Bu}), 19.2(\mathrm{C}), 1.7\left(\mathrm{CH}_{2}-\mathrm{I}\right)$; HRMS-ESI $m / z$ calcd for $\mathrm{C}_{30} \mathrm{H}_{44} \mathrm{INO}_{5} \mathrm{SiNa}[\mathrm{M}+\mathrm{Na}]^{+}: 676.1931$, found: 676.1905.

tert-Butyl $N$-benzyl- $N$-[(1S)-1-[(tert-butyldiphenylsilyloxy)methyl]-3-iodopropyl]carbamate (16c). A stirred solution of $(S)-15 c(0.885 \mathrm{~g}, 1.66 \mathrm{mmol})$ in anhydrous tetrahydrofuran $(10 \mathrm{~mL})$ 
at $0{ }^{\circ} \mathrm{C}$ and under argon atmosphere was successively treated with triethylamine $(348 \mu \mathrm{L}, 2.48$ mmol $)$, 4-dimethylaminopyridine $(0.040 \mathrm{~g}, 0.33 \mathrm{mmol})$ and methanesulfonyl chloride $(260 \mu \mathrm{L}$, $3.32 \mathrm{mmol}$ ). After $20 \mathrm{~min}$, the reaction mixture was diluted with diethyl ether. The organic layer was washed with water followed by brine and dried $\left(\mathrm{MgSO}_{4}\right)$. After filtration and concentration, the crude mesylate of $15 \mathrm{c}$ thus obtained was diluted in acetone $(25 \mathrm{~mL})$. Sodium iodide $(4.97 \mathrm{~g}$, $33.2 \mathrm{mmol}$ ) was added and the reaction mixture was stirred at $20^{\circ} \mathrm{C}$ for $12 \mathrm{~h}$. The precipitate was dissolved by adjunction of water and the crude mixture was extracted with diethyl ether. The organic layer was washed twice with $10 \%$ aqueous sodium thiosulfate solution, water and brine. After drying $\left(\mathrm{MgSO}_{4}\right)$, the resulting solution was filtered and the solvent was evaporated under vacuo. The residue was purified by column chromatography (diethyl ether/pentane, 1:9) affording 16c as a yellow oil (755 mg, 71\%). Compound 16c was detected in the NMR spectra at ambient temperature as a mixture of two rotamers $a$ and $b . R_{f}=0.56$ (EtOAc/cyclohexane, 1/19); $[\alpha]_{\mathrm{D}}{ }^{25}=-14.4\left(\mathrm{c} 1.13, \mathrm{CHCl}_{3}\right)$; IR (neat) $v_{\max } 1694 ;{ }^{1} \mathrm{H}$ NMR $\left(\mathrm{CDCl}_{3}\right) \delta 7.60-7.21(\mathrm{~m}, 15 \mathrm{H}, \mathrm{H}-$ Ar $a$ and $b), 4.47\left(\mathrm{~m}, 2 \mathrm{H}, \mathrm{CH}_{2}-\mathrm{Ph}, a\right), 4.41\left(\mathrm{~m}, 2 \mathrm{H}, \mathrm{CH}_{2}-\mathrm{Ph}, b\right), 4.07(\mathrm{~m}, 1 \mathrm{H}, \mathrm{CH}, a), 3.90(\mathrm{~m}$, $1 \mathrm{H}, \mathrm{CH}, b), 3.71\left(\mathrm{~m}, 2 \mathrm{H}, \mathrm{CH}_{2}-\mathrm{O}, b\right), 3.60\left(\mathrm{~m}, 2 \mathrm{H}, \mathrm{CH}_{2}-\mathrm{O}, a\right), 2.96\left(\mathrm{~m}, 2 \mathrm{H}, \mathrm{CH}_{2}-\mathrm{I}, a\right), 2.82(\mathrm{~m}$, $\left.2 \mathrm{H}, \mathrm{CH}_{2}-\mathrm{I}, b\right), 2.14\left(\mathrm{~m}, 2 \mathrm{H}, \mathrm{CH}_{2}, b\right), 2.03\left(\mathrm{~m}, 2 \mathrm{H}, \mathrm{CH}_{2}, a\right), 1.44(\mathrm{~s}, 9 \mathrm{H}, t-\mathrm{Bu}, \mathrm{a}), 1.39(\mathrm{~s}, 9 \mathrm{H}, t-$ $\mathrm{Bu}, b), 1.03(\mathrm{~s}, 9 \mathrm{H}, t \mathrm{Bu}, a$ and $b) ;{ }^{13} \mathrm{C} \mathrm{NMR}\left(\mathrm{CDCl}_{3}\right) \delta 156.0\left(\mathrm{CO}_{2} t \mathrm{Bu}, a\right), 155.4\left(\mathrm{CO}_{2} t \mathrm{Bu}, b\right)$, 139.4 (C-Ar, b), 139.0 (C-Ar, $a$ ), 135.4 (C-Ar, $a$ and $b$ ), 133.0 (C-Ar, $a$ and $b$ ), 129.6 (C-Ar, $a$ and $b$ ), 128.3 (C-Ar, $a$ and $b$ ), 127.6 (C-Ar, $a$ and $b), 127.0$ (C-Ar), 80.1 (C, $a), 79.7(\mathrm{C}, b), 64.5$ $\left(\mathrm{CH}_{2}-\mathrm{O}, a\right), 64.1\left(\mathrm{CH}_{2}-\mathrm{O}, b\right), 59.3(\mathrm{CH}, a$ and $b), 49.6\left(\mathrm{CH}_{2}-\mathrm{Ph}, a\right.$ and $\left.b\right), 34.4\left(\mathrm{CH}_{2}, a\right), 34.1$ $\left(\mathrm{CH}_{2}, b\right), 28.3(t-\mathrm{Bu}, a$ and $b), 26.7(t-\mathrm{Bu}, a$ and $b), 19.0(\mathrm{C}, a$ and $b), 2.4\left(\mathrm{CH}_{2}-\mathrm{I}, a\right.$ and $\left.b\right)$; HRMS-ESI $m / z$ calcd for $\mathrm{C}_{32} \mathrm{H}_{42} \mathrm{INO}_{3} \mathrm{Si}[\mathrm{M}+\mathrm{Na}]^{+}: 666.1876$, found: 666.1845 .

tert-Butyl $\quad N$-[(4S)-4-(2-iodoethyl)-2,2-dimethyl-1,3-oxazolidin-3-yl]carboxylate (16d). Compound 16d was prepared in toluene following the general procedure for the preparation of iodides. Yellow foam $(0.297 \mathrm{~g}, 82 \%) ; m p 45-47{ }^{\circ} \mathrm{C}$ (pentane); $R_{f} 0.60$ (EtOAc/cyclohexane, 3/7); $[\alpha]_{\mathrm{D}}{ }^{25}=+17.8\left(\mathrm{c} 0.1, \mathrm{CHCl}_{3}\right)$ (for its $(R)$-enantiomer lit. ${ }^{19}[\alpha]_{\mathrm{D}}{ }^{25}=-16.2\left(\mathrm{c} 2.6, \mathrm{CHCl}_{3}\right)$ ); IR

$(\mathrm{KBr}) v_{\max } 1698 ;{ }^{1} \mathrm{H}$ NMR $\left(\mathrm{C}_{6} \mathrm{D}_{6}, 70{ }^{\circ} \mathrm{C}\right) \delta 3.69(\mathrm{~m}, 1 \mathrm{H}, \mathrm{CH}), 3.52(\mathrm{ddt}, J=9.0,6.0,1.0 \mathrm{~Hz}, 1 \mathrm{H}$, $\left.\mathrm{CH}_{2}-\mathrm{O}\right), 3.29$ (dt, $\left.J=9.0,1.5 \mathrm{~Hz}, 1 \mathrm{H}, \mathrm{CH}_{2}-\mathrm{O}\right), 2.86\left(\mathrm{td}, J=9.0,5.5 \mathrm{~Hz}, 1 \mathrm{H}, \mathrm{CH}_{2}-\mathrm{I}\right), 2.74(\mathrm{dt}, J=$ 9.0, $\left.8.0 \mathrm{~Hz}, 1 \mathrm{H}, \mathrm{CH}_{2}-\mathrm{I}\right), 2.15\left(\mathrm{~m}, 1 \mathrm{H}, \mathrm{CH}_{2}\right), 1.85$ (dtd, $\left.J=14.0,8.0,6.0 \mathrm{~Hz}, 1 \mathrm{H}, \mathrm{CH}_{2}\right), 1.55(\mathrm{~s}$, $\left.3 \mathrm{H}, \mathrm{CH}_{3}\right), 1.43\left(\mathrm{~s}, 3 \mathrm{H}, \mathrm{CH}_{3}\right), 1.39(\mathrm{~s}, 9 \mathrm{H}, t-\mathrm{Bu}) ;{ }^{13} \mathrm{C} \mathrm{NMR}\left(\mathrm{C}_{6} \mathrm{D}_{6}, 70{ }^{\circ} \mathrm{C}\right) \delta 150.9\left(\mathrm{CO}_{2} t \mathrm{Bu}\right), 94.0$ $(\mathrm{C}), 79.7(\mathrm{C}), 66.7\left(\mathrm{CH}_{2}-\mathrm{O}\right), 58.6(\mathrm{CH}), 38.2\left(\mathrm{CH}_{2}\right), 28.6(t-\mathrm{Bu}), 27.3\left(\mathrm{CH}_{3}\right), 24.0\left(\mathrm{CH}_{3}\right), 0.3$ $\left(\mathrm{CH}_{2}-\mathrm{I}\right)$; MS-ESI m/z $378\left([\mathrm{M}+\mathrm{Na}]^{+}, 100\right), 338$ (34), 33 (21).

\section{References}

1. (a) Troast, D. M.; Yuan, J.; Porco Jr., J. A. Adv. Synth. Catal. 2008, 350, 1701. (b) Reymond, S.; Cossy, J. Tetrahedron 2007, 63, 5918.

2. Dave, R.; Sasaki, N. A. Tetrahedron: Asymmetry 2006, 17, 388.

3. Ostendorf, M.; Dijkink, J.; Rutjes, F. P. J. T. ; Hiemstra, H. Eur. J. Org. Chem. 2000, 115. 
4. Faulkner, D. J. Nat. Prod. Prep. 1984, 25.

5. (a) For a pioneering work see Enders, D.; Gatzweiler, W.; Dederichs, E. Tetrahedron 1990, 46, 4757. (b) Crimmins, M. T.; Rafferty, S. W. Tetrahedron Lett 1996, 37, 5649. (c) Tursun, A.; Canet, I.; Aboab, B.; Sinibaldi, M.-E. Tetrahedron Lett. 2005, 46, 2291. (d) Tursun, A.; Aboab, B.; Martin, A.-S.; Sinibaldi, M.-E.; Canet, I. Synlett 2005, 15, 2397.

6. (a) Hirth, G.; Walther, W. Helv. Chim. Acta 1985, 68, 1863. (b) Mukaiyama, T.; Shiina, I.; Iwadare, H.; Saitoh, M.; Nishimura, T.; Ohkawa, N.; Sakoh, H.; Nishimura, K.; Tani, Y.-I.; Hasegawa, M.; Yamada, K.; Saitoh, K. Chem. Eur. J. 1999, 5, 121. (c) For spectroscopic data of $(S)$-2 enantiomer see Eichelberger, U.; Mansourova, M.; Hennig, L.; Findeisen, M.; Giesa, S.; Müller, D.; Welzel, P. Tetrahedron 2001, 57, 9737.

7. (2R)-Alcohol 3 possessed spectral data consistent with those reported in the literature by Green, M. E.; Rech, J. C.; Floreancig, P. E. Org. Lett. 2005, 7, 4117.

8. Goubert, M.; Toupet, L.; Sinibaldi, M.-E.; Canet, I.; Tetrahedron 2007, 63, 8255.

9. For the spectroscopic data of (S)-5a see Peters, U.; Bankova, W.; Welzel, P. Tetrahedron 1987, 43, 3803.

10. For its (S)-isomer see (a) Barco, A.; Benetti, S.; Spallutto, G. J. Org. Chem. 1992, 57, 6279. (b) Chalker, J. M; Yang, A.; Deng, K.; Cohen, T. Org. Lett., 2007, 9, 3825.

11. For preparation and spectroscopic data for its (S)-isomer see: (a) McNulty, J.; Still, I. W. J. Synth. Commun. 1992, 22, 979. (b) Vaswani, R. G.; Chamberlin, A. R. J. Org. Chem. 2008, 73, 1661 .

12. (a) Dave, R.; Sasaki, N. A. Org. Lett. 2004, 6, 15. (b) Dondoni, A.; Perrone, D.; Merino, P. J. Org. Chem. 1995, 60, 8074.

13. (a) Williams, L.; Zhang, Z.; Shao, F.; Carroll, P. J.; Joullié, M. M. Tetrahedron 1996, 52, 11673. (b) Garner, P.; Park, J. M. J. Org. Chem. 1987, 52, 2361.

14. For another method of preparation and spectroscopic data of $(S)-10 b$ see: Cong, X.; Hu, F.; Liu, K.-G.; Liao, Q.-J.; Yao, Z.-J. J. Org. Chem. 2005, 70, 4516.

15. Kadota, I., Saya, S.; Yamamoto, Y. Heterocycles 1997, 46, 335.

16. For another method of preparation and partial spectroscopic data of $(S)-\mathbf{1 2}$ see Brinkmann, T.; Gilg, A.; Hamm, A.; Lüsch, H.; Morbach, G.; Uzar, H. C. Tetrahedron: Asymmetry 2000, 11,3827 .

17. Markidis, T.; Kokotos, G. J. Org. Chem. 2001, 66, 1919.

18. (a) For another method of preparation of $(S)$-15d see Hou, D.-R.; Reibenspies, J. H.; Burgess, K. J. Org. Chem. 2001, 66, 206. (b) Paintner, F. F.; Allmendinger, L.; Bauschke, G.; Klemann, P. Org. Lett. 2005, 7, 1423. (c) Ksander, G. M.; DeJesus, R.; Yuan, A.; Ghai, R. D.; Trapani, A.; McMartin, C.; Bohacek, R. J. Med. Chem. 1997, 40, 495.

19. Campbell, A. D.; Raynham, T. M.; Taylor, R. J. K. Tetrahedron Lett. 1999, 40, 5263. 